\title{
Impact of Surface Flux Formulations and Geostrophic Forcing on Large-Eddy Simulations of Diurnal Atmospheric Boundary Layer Flow
}

\author{
VIJAYANT KUMAR \\ Department of Geography and Environmental Engineering, The Johns Hopkins University, Baltimore, Maryland \\ GunILLA SVENSSON \\ Department of Meteorology, Stockholm University, Stockholm, Sweden \\ A. A. M. Holtslag \\ Meteorology and Air Quality Group, Wageningen University, Wageningen, Netherlands \\ Charles Meneveau \\ Department of Mechanical Engineering, The Johns Hopkins University, Baltimore, Maryland \\ MARC B. PARLANGE \\ School of Architecture, Civil and Environmental Engineering, Ecole Polytechnique Fédérale de Lausanne, \\ Lausanne, Switzerland
}

(Manuscript received 29 October 2008, in final form 29 October 2009)

\begin{abstract}
The impact of surface flux boundary conditions and geostrophic forcing on multiday evolution of flow in the atmospheric boundary layer (ABL) was assessed using large-eddy simulations (LES). The LES investigations included several combinations of surface boundary conditions (temperature and heat flux) and geostrophic forcing (constant, time varying, time and height varying). The setup was based on ABL characteristics observed during a selected period of the Cooperative Atmosphere-Surface Exchange Study-1999 (CASES-99) campaign. The LES cases driven by a constant geostrophic wind achieved the best agreement with the CASES-99 observations specifically in terms of daytime surface fluxes and daytime and nighttime profiles. However, the nighttime fluxes were significantly overestimated. The LES cases with the surface temperature boundary condition and driven by a time- and height-varying geostrophic forcing showed improved agreement with the observed nighttime fluxes, but there was less agreement with other observations (e.g., daytime profiles). In terms of the surface boundary condition, the LES cases driven by either surface temperature or heat fluxes produced similar trends in terms of the daytime profiles and comparisons with data from soundings. However, in reproducing the fluxes and nighttime profiles, the agreement was better with imposed temperature because of its ability to interact dynamically with the air temperature field. Therefore, it is concluded that surface temperature boundary condition is better suited for simulations of temporally evolving ABL flow as in the diurnal evolution of the ABL.
\end{abstract}

\section{Introduction and background}

The diurnal cycle of solar and atmospheric forcings has profound implications on the mass, momentum, and energy exchanges occurring in the atmospheric boundary

Corresponding author address: Vijayant Kumar, 4701 Monterey Oaks Blvd., Apt. 2310, Austin, TX 78749.

E-mail: vijayant@gmail.com layer (ABL). Since the primary agent responsible for the vertical transport and mixing in the $\mathrm{ABL}$ is atmospheric turbulence, major modeling efforts have been directed toward a better understanding and representation of the diurnal structure of atmospheric turbulence.

A primary objective inherent to most ABL modeling efforts is improving the accuracy of turbulence parameterizations being used in large-scale numerical weather prediction and climate models. To be specific, the 
parameterization of stable boundary layers (SBLs) has been the focus of recent intercomparison studies using large-eddy simulation (LES) and single-column models (SCM) (Beare et al. 2006; Cuxart et al. 2006). To account for boundary layer turbulence, the SCMs rely on turbulence parameterizations such as first-order, turbulent kinetic energy (TKE), and other higher-order schemes. The inherent attractiveness of SCMs owing to a high computational efficiency is tempered by the fact that the use of different turbulence parameterizations (e.g., first order, TKE, higher order) in SCMs leads to a large variability in forecasts of land temperature during winter, mixing (Cuxart et al. 2006), and surface drag in stable boundary layers (Beljaars and Viterbo 1998).

The LES technique, on the other hand, is less affected by parameterization issues because it explicitly simulates the large-scale structures resolved by the computational grid while the effects of the small, unresolved scales are parameterized using a subgrid-scale (SGS) model. LES has emerged as a popular research tool in simulation of ABL flows and has been used for simulations of neutral, convective, and stable ABLs (Andrén et al. 1994; Kosović and Curry 2000; Moeng 1984; Nieuwstadt et al. 1991). Several LES studies have pointed out discrepancies (e.g., strong attenuation of velocity and temperature spectra resulting in departure from the expected inertial scaling) associated with the use of conventional SGS models in simulations of convective (Schmidt and Schumann 1989; Nieuwstadt et al. 1991) boundary layers. In a similar way, numerical instabilities and departures from inertial-range spectral scalings have also plagued LES investigations of the stable boundary layer (Beare and MacVean 2004; Derbyshire 1999; Saiki et al. 2000; Mason and Derbyshire 1990), arising mainly from the smaller turbulence structures in the stable boundary layer. Note that the issue of accurate SGS models becomes even more paramount in the stable boundary layer because the SGS terms contain a significant amount of the total turbulence kinetic energy.

Recent attempts through new-generation variants of the dynamic models have shown good promise. Notable is that the Lagrangian averaged scale-dependent dynamic model (Bou-Zeid et al. 2005) has demonstrated accurate reproduction of characteristic features and inertial-range spectral scalings in LES of quasi-steady convective and stable boundary layers (Kleissl et al. 2006). Another variant, the locally averaged scale-dependent dynamic model (Basu and Porte-Agel 2006), has also been applied to LES of stable boundary layers, achieving good comparison to the results from the first Global Energy and Water Cycle Experiment (GEWEX) Atmospheric Boundary Layer Study (GABLS) LES intercomparison (Beare et al. 2006).
The focus of the majority of the above-mentioned LES studies has been subgrid-scale modeling, analyzing characteristic features of quasi-steady ABLs, and/or performance testing of existing parameterizations. The LES is inherently superior to SCMs in terms of resolving the full three-dimensional structure of the turbulent flow field, but the quality of both LES and SCM results is still heavily dependent on the surface flux boundary conditions and the imposed geostrophic wind. Therefore, to develop LES tools capable of handling nonstationary inputs and producing high-resolution results for comparison with surface-layer observations, investigations of the impact of variable boundary conditions and geostrophic forcing are imperative.

An LES code and the accompanying SGS model for such investigations should be capable of 1) dynamic adjustment to change in atmospheric stability in a diurnal cycle and 2) producing correct turbulence statistics representative of the ABL dynamics. The Johns Hopkins University LES code embedded with a new-generation Lagrangian scale-dependent model has recently demonstrated such capabilities through high-resolution LES investigations of the diurnal ABL and surface-layer comparisons with the 2000 Horizontal Array Turbulence Study (HATS2000) campaign (Kumar et al. 2006). This LES code yields predictions that correctly exhibit wellknown and widely observed spectral scalings in neutral, convective, and stable boundary layers (Porté-Agel et al. 2000; Bou-Zeid et al. 2005; Kleissl et al. 2006). The code has also been validated with meteorological observations and particle image velocimetry for an LES study of pollen transport in canopies (Yue et al. 2007, 2008; Chamecki et al. 2009).

The results of Kleissl et al. (2006) demonstrated characteristic features of the diurnal ABL, such as the evolution of the low-level jet (LLJ), and yielded good qualitative agreement with observations in both convective and stable regimes. This study, however, was based on highly idealized geostrophic forcing and a surface heat flux boundary condition. Thus, to further ascertain the capabilities of the code, it should be tested using "realistic" nonstationary forcing and surface boundary conditions. This implies that we need a dataset from which both the required surface boundary conditions and geostrophic forcing conditions can be obtained for a multiday duration. In addition, to assess the accuracy of LES predictions, such an observational database should ideally contain vertical profiles of velocity components, fluxes, and other relevant quantities for the same time period.

Keeping these requirements in mind, the well-known Cooperative Atmosphere-Surface Exchange Study-1999 (CASES-99) campaign would serve well as a setup for the LES of multiday evolution of the ABL. GABLS is 
TABLE 1. LES case details for the six LES simulations. The header contains the nomenclature assigned to refer to each of the six LES cases. The first two rows respectively indicate the nature of the underlying imposed boundary condition for surface flux calculation and the nature of the imposed geostrophic forcing. The remaining rows contain simulation parameters that apply to all of the LES cases.

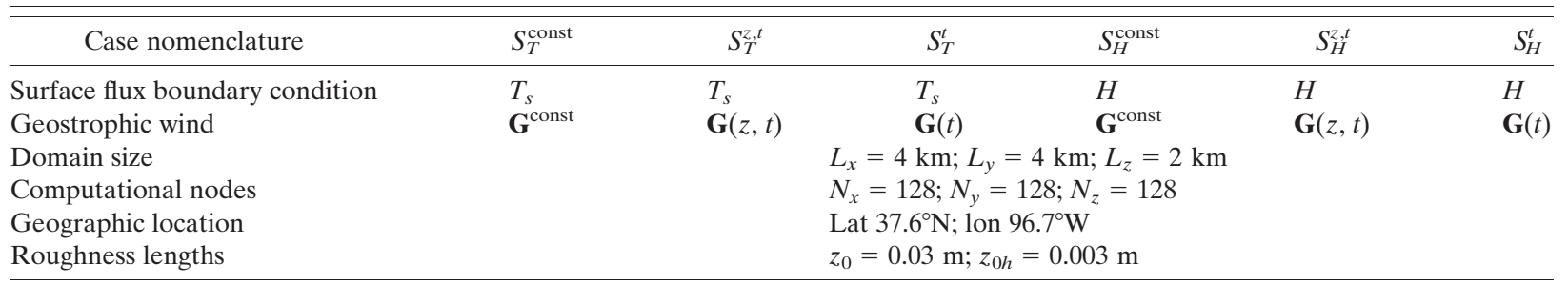

a project under the aegis of GEWEX, aimed at improving the representation of the ABL in climate models. An SCM intercomparison of the multiday evolution of the ABL based on CASES-99 observations (Poulos et al. 2002) was recently performed as part of the GABLS2 initiative (http://www.gewex.org/gabls.htm). Considering the direct relevance of this SCM intercomparison to our LES studies, we have used the underlying setup from the SCM intercomparison as a guiding framework for the LES simulations presented in this work. Note that the SCMs have increasingly relied on LES as a benchmarking/ reference tool for testing and developing new regional/ GCM turbulence parameterizations.

The goal of the current LES study therefore is to integrate CASES-99 observations into the LES setup and to perform a suite of simulations using several combinations of geostrophic forcing and surface flux boundary conditions. As such, we built on related studies by Steeneveld et al. (2006) and Holtslag et al. (2007), in which an SCM is used for comparison with the observations and to analyze the role of surface temperature feedbacks. Using the setup and findings of the LES of this study, we are also able to compare the results with the ensemble mean of a variety of SCMs for GABLS2 (Svensson and Holtslag 2006, 2007). The results obtained from these simulations will be analyzed to illustrate the impact of geostrophic forcing and surface boundary conditions and may be used as a benchmark for SCM investigations.

The simulation setup for the LES investigations is explained in detail in section 2. Simulations with a constant geostrophic forcing and forced by surface temperature and surface heat flux boundary conditions are presented in section 3. Next, simulations governed by time-varying and both time- and height-varying geostrophic forcing in conjunction with the two surface boundary conditions are presented (section 4), resulting in a total of four additional simulations. The six resulting cases, as summarized in Table 1, thus represent various combinations of the two surface flux boundary conditions and three types of geostrophic forcing. Deductions pertaining to the impact of geostrophic forcing and surface heat flux boundary condition resulting from an analysis of the various simulation results are presented in section 5 .

\section{LES setup}

The observations from the CASES-99 campaign have been utilized to obtain surface boundary conditions including surface temperature and surface heat flux. The geostrophic forcing is derived from a mesoscale simulation using the Coupled Ocean-Atmosphere Mesoscale Prediction System (Hodur 1997) run. The LES domain size is $4000 \mathrm{~m} \times 4000 \mathrm{~m} \times 2000 \mathrm{~m}$. With 128 computational nodes in each direction, the resolutions are $\Delta x, \Delta y$, and $\Delta z=31.25,31.25$, and $15.625 \mathrm{~m}$, respectively. Note that with the staggered grid formulation the first computational node is at a height of $\Delta z / 2=$ $7.813 \mathrm{~m}$. As mentioned in the preceding sections, the LES uses the code described in Albertson and Parlange (1999a,b) with subsequent modifications as described in Bou-Zeid et al. (2005) and Kumar et al. (2006). The SGS momentum and heat flux formulations are of the eddyviscosity type and are modeled using a combination of a Lagrangian dynamic scale-dependent SGS model and an imposed constant SGS Prandtl number $\left(\operatorname{Pr}_{\text {sgs }}=0.4\right)$. The momentum roughness length $z_{0 m}$ and scalar roughness length $z_{0 h}$ are imposed as 0.03 and $0.003 \mathrm{~m}$, respectively.

The lower boundary condition is formulated using the Monin-Obukhov similarity (MOS) theory wherein the instantaneous surface stress is represented as a function of instantaneous horizontal velocities $\tilde{u}$ and $\tilde{v}$ at the first grid point $(d z / 2)$ above the surface:

$$
\begin{aligned}
& \tau_{x z}=-u_{*}^{2}\left(\frac{\hat{\tilde{u}}}{\sqrt{\hat{\tilde{u}}^{2}+\hat{\tilde{v}}^{2}}}\right) \text { and } \\
& \tau_{y z}=-u_{*}^{2}\left(\frac{\hat{\tilde{v}}}{\sqrt{\hat{\tilde{u}}^{2}+\hat{\tilde{v}}^{2}}}\right),
\end{aligned}
$$

where $u_{*}$ is the friction velocity computed as 


$$
u_{*}=\frac{\kappa \sqrt{\hat{\tilde{u}}^{2}+\hat{\tilde{v}}^{2}}}{\ln \left(\frac{z}{z_{0}}\right)-\psi_{m}\left(\frac{z}{L}\right)} .
$$

Here, $\kappa$ is the von Kármán constant, $z_{0}$ is the momentum roughness length, and $L$ is the Obukhov length. Note that in Eqs. (1)-(3), the instantaneous velocities $\tilde{u}$ and $\tilde{v}$ are explicitly test filtered at scale $2 \Delta$ as represented by an overlying caret symbol. The reasoning for the test filtering is that the MOS theory for the mean flow is applied here in a strictly local sense and the test filtering therefore serves to reduce inaccuracy associated with the small-scale fluctuations while still preserving largescale variations (Bou-Zeid et al. 2005).

The $\psi_{m}$ functions introduced in Eq. (3) are defined as

$$
\psi_{m}(z / L)=\int_{z_{0} / L}^{z / L}\left[1-\phi_{m}(x)\right] d x / x
$$

The Monin-Obukhov stability correction functions for nondimensional gradients $\phi_{m}\left[=\left(k z / u_{*}\right)(d U / d z)\right]$ and $\phi_{h}$ $\left[=\left(-k z u_{*} / \overline{w^{\prime} \theta_{s}^{\prime}}\right)(d \theta / d z)\right]$ are needed for the bottom boundary condition. For unstable conditions, the formulation of $\phi_{m}$ and $\phi_{h}$ is based on Högström (1987). For the stable regimes, the simple formulations for $\phi_{m}$ and $\phi_{h}$ are of type $\alpha+\beta(z / L)$ (where $\alpha$ and $\beta$ are constants) and are not consistent with observations when $z / L$ is greater than 0.8 (Hicks 1976; Holtslag and De Bruin 1988), and hence the modified formulation of Cheng and Brutsaert (2005) is used. Thus, $\phi_{m}$ and $\phi_{h}$ are given by

$$
\begin{aligned}
& \phi_{m}= \begin{cases}\left(1-15.2 \frac{z}{L}\right)^{-0.25} & \text { for } z / L<0 \\
1+6.1 \frac{z}{L}+\left(\frac{z}{L}\right)^{2.5}\left[1+\left(\frac{z}{L}\right)^{2.5}\right]^{(-1+1 / 2.5)} & \text { for } z / L>0 \\
\frac{z}{L}+\left[1+\left(\frac{z}{L}\right)^{2.5}\right]^{1 / 2.5} & \text { and }\end{cases} \\
& \phi_{h}= \begin{cases}\left(1-15.2 \frac{z}{L}\right)^{-0.5} & \text { for } z / L<0 \\
1+5.3 \frac{z}{L}+\left(\frac{z}{L}\right)^{1.1}\left[1+\left(\frac{z}{L}\right)^{1.1}\right]^{(-1+1 / 1.1)} & \text { for } z / L>0 \\
\frac{z}{L}+\left[1+\left(\frac{z}{L}\right)^{1.1}\right]^{1 / 1.1} & \end{cases}
\end{aligned}
$$

The code has the capability either to impose surface heat fluxes directly or to compute them by applying MOS theory relating the surface temperature and air temperature at the first computational node. We will refer to these two boundary conditions respectively as $H$ and $T_{s}$ in correspondence to the nature of the imposed variable.

With an imposed surface temperature boundary condition $T_{s}$, the surface sensible heat fluxes $\overline{\tilde{w}^{\prime} \tilde{\theta}_{s}^{\prime}}$ are calculated in the LES using the MOS relationship between the imposed surface temperature $T_{s}$ and the temperature of the overlying atmosphere at the first vertical node above the surface $\tilde{\theta}(\Delta z / 2)$, as represented by the equation

$$
\overline{\tilde{w}^{\prime} \tilde{\theta}_{s}^{\prime}}=\kappa \frac{u_{*} T_{s}-u_{*} \tilde{\theta}(0.5 d z)}{\log \left(0.5 d z / z_{h}\right)-\psi_{h}(0.5 d z / L)},
$$

with the MOS function $\psi_{h}$ given by Eq. (7):

$$
\psi_{h}\left(\frac{z}{L}\right)=\int_{z_{0} / L}^{z / L}\left[1-\phi_{h}(x)\right] \frac{d x}{x} .
$$

\section{LES using the GABLS SCM intercomparison setup}

The GABLS SCM intercomparison setup (Svensson and Holtslag 2007) of the diurnal cycle serves as the reference for the LES investigation of a multiday evolution of the diurnal ABL. The simulation is initialized at 1600 LT 22 October 1999 with a constant geostrophic wind velocity profile $\left(U_{g}, V_{g}\right)=(3,-9) \mathrm{m} \mathrm{s}^{-1}$ and a mean temperature profile that are presented in Fig. 1. Random velocity and temperature values scaled according to the specified mean TKE profile $[\bar{e}(z)=0.5(1-z / 800)$ for $z \leq 800 \mathrm{~m} ; 0$ otherwise] are added to the mean velocity $(u, v$, and $w)$ profiles to initialize the turbulence. The initialization method can be compactly represented for any variable $a(x, y, z)$ as

$$
\begin{aligned}
a(x, y, z) & =\bar{a}(z)+\frac{\sigma_{\bar{e}(z)}}{\sigma_{\text {rand }}}\left[\operatorname{rand}_{(0,1)}-0.5\right] \\
& =\bar{a}(z)+\frac{\sqrt{\frac{2}{3} \bar{e}(z)}}{0.289}\left[\operatorname{rand}_{(-0.5,0.5)}\right],
\end{aligned}
$$



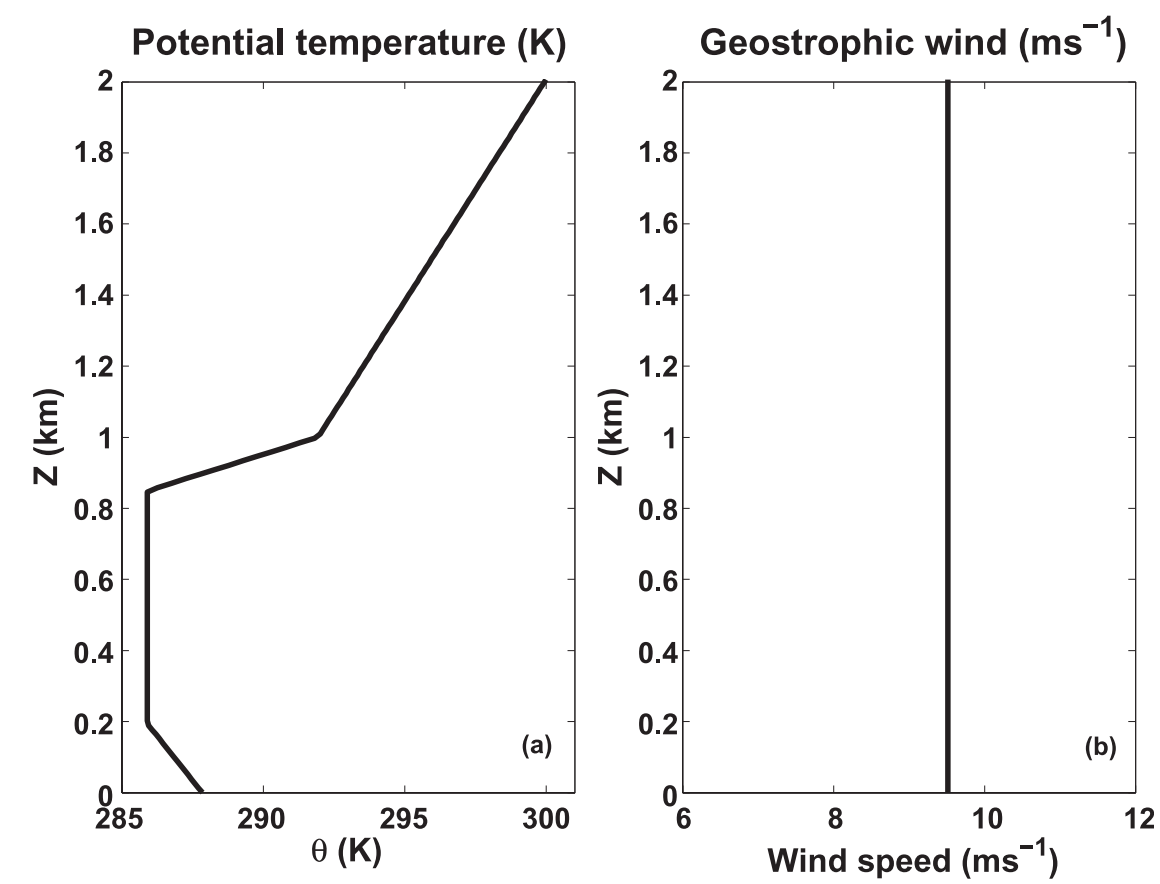

FIG. 1. Vertical profile of (a) potential temperature and (b) geostrophic wind used for initialization of LES.

where $\bar{a}(z)=U_{g}, V_{g}, 0$ for $u, v$, and $w$, respectively, and $\bar{e}(z)$ is as specified above (see Tables 2-5 for detailed information regarding the initial profiles and temporal forcing). A large-scale synoptic divergence or subsidence $w_{s}$ is applied starting at hour 40 of the simulation period and is prescribed as $w_{s}=-0.005 z / 1000 \mathrm{~m} \mathrm{~s}^{-1}$ for $z \leq$ $1000 \mathrm{~m}$ and $w_{s}=-0.005 \mathrm{~m} \mathrm{~s}^{-1}$ for $z>1000 \mathrm{~m}$. Note that, unlike the SCM setup, no evolution of humidity is simulated in the presented LES investigations.

The simulation is run for a total time period of $52 \mathrm{~h}$, terminating at 2000 LT 24 October 1999. The temporal evolution of the surface temperature $T_{S}$ is derived as a simplified fit to the CASES-99 observations (henceforth referred to as C99) as shown in Fig. 2 (top panel). For more details about the case and the SCM results, see G. Svensson et al. (2010, manuscript submitted to Bound.-Layer Meteor.).

TABLE 2. Simulation parameters: initial vertical profile of potential temperature and horizontal components of velocity.

\begin{tabular}{rcc}
\hline \hline $\begin{array}{c}\text { Height } \\
z(\mathrm{~m})\end{array}$ & $\begin{array}{c}\text { Potential } \\
\text { temperature } \theta(\mathrm{K})\end{array}$ & $\begin{array}{c}\text { Velocity components } \\
(u, v)\left(\mathrm{m} \mathrm{s}^{-1}\right)\end{array}$ \\
\hline 0 & 288 & $(0,0)$ \\
200 & 286 & $(3,-9)$ \\
850 & 286 & $(3,-9)$ \\
900 & 288 & $(3,-9)$ \\
1000 & 292 & $(3,-9)$ \\
2000 & 300 & $(3,-9)$ \\
\hline
\end{tabular}

We will denote this simulation as $S_{T}^{\text {const }}$. The simulation $S_{T}^{\text {const }}$ is forced by a constant geostrophic forcing given by

$$
\begin{gathered}
\mathbf{G}^{\text {const }} \equiv\left(U_{g}, V_{g}\right)=(3,-9) \mathrm{m} \mathrm{s}^{-1} ; \\
\left(\left|\mathbf{G}^{\text {const }}\right|=\sqrt{U_{g}^{2}+V_{g}^{2}}=9.5 \mathrm{~m} \mathrm{~s}^{-1}\right)
\end{gathered}
$$

(Fig. 3a). Figure 3a also demarcates the time periods corresponding to 22, 23, and 24 October 1999, which will be henceforth referred to in the text as day 1 , day 2 , and day 3 , respectively. Note that the bottom panel of Fig. 2 shows the time series of $\mathrm{C} 99$ surface heat flux observations and a simplified fit for use as an LES boundary condition. We will come back to this again in section 3b.

\section{a. Comparison between LES and SCM runs}

This section presents comparisons between SCM and LES results with the $S_{T}^{\text {const }}$ setup. For the purposes of this study, the SCM results will be represented by the mean of all of the SCM models. The spread associated with the various SCM codes involved in the intercomparison

TABLE 3. As in Table 2, but for TKE.

\begin{tabular}{cc}
\hline \hline Height $z(\mathrm{~m})$ & TKE \\
\hline$z \leq 800 \mathrm{~m}$ & $0.5(1-z / 800)$ \\
$z>800 \mathrm{~m}$ & 0 \\
\hline
\end{tabular}


TABLE 4. Simulation parameters: temporal behavior (evolution) of surface boundary condition for LES cases with prescribed skin temperature $T_{s}\left({ }^{\circ} \mathrm{C}\right)$. Note that local time $t$ represents total elapsed time past the simulation onset point (i.e., 1600 LT). For instance, at the end of simulation period of $52 \mathrm{~h}, t=68$.

\begin{tabular}{lc}
\hline \hline Local time $t$ & Skin temperature $T_{s}\left({ }^{\circ} \mathrm{C}\right)$ \\
\hline$t \leq 17.4$ & $-10-25 \cos (0.22 t+0.2)$ \\
$17.4<t \leq 30$ & $0.54 t+15.2$ \\
$30<t \leq 41.9$ & $-7-25 \cos (0.21 t+1.8)$ \\
$41.9<t \leq 53.3$ & $-0.37 t+18$ \\
$53.3<t \leq 65.6$ & $-4-25 \cos (0.22 t+2.5)$ \\
$t>65.6$ & 4.4 \\
\hline
\end{tabular}

(defined as the envelope encompassing all the SCM model results) is represented by the error bars. In all three panels in Fig. 4, LES and SCM results exhibit mutually consistent trends, respectively, for the surface layer variables (i.e., friction velocity $u_{*}, 10-\mathrm{m}$ horizontal wind speed $U_{10}$, and sensible heat flux $\left.\overline{\tilde{w}^{\prime} \tilde{\theta}_{s}^{\prime}}\right)$. The LES results consistently fall within the data spread of the SCM results. It is encouraging that even with a coarser near-surface resolution, the surface layer results from LES capture the diurnal trends well. Note that the main purpose of presenting SCM results here was to demonstrate the consistent agreement displayed by the SCM models with the LES results. Our prime objective, however, is on assessing the performance of
TABLE 5. As in Table 4, but for LES cases with prescribed surface heat flux $H\left(\mathrm{~K} \mathrm{~m} \mathrm{~s}^{-1}\right)$.

\begin{tabular}{ll}
\hline \multicolumn{1}{c}{ Local time $t$} & \multicolumn{1}{c}{$\begin{array}{c}\text { Surface heat flux } \\
H\left(\mathrm{~K} \mathrm{~m} \mathrm{~s}^{-1}\right)\end{array}$} \\
\hline$t \leq 16.5$ & $-0.0338+0.2 \cos (0.44 t+0.5)$ \\
$16.5<t \leq 30.5$ & -0.015 \\
$30.5<t \leq 40.5$ & $-0.2094-0.395 \cos (0.2098 t+1.97)$ \\
$40.5<t \leq 54.5$ & -0.0102 \\
$54.5<t \leq 65$ & $-0.471-0.656 \cos (0.15107 t+0.4)$ \\
$t>65$ & $(-0.2286 t+13.46) / 100$ \\
\hline
\end{tabular}

LES vis-à-vis the C99 observations, and hence the subsequent portions of this article will focus exclusively on comparisons between LES results and C99 observations.

\section{b. Comparison of LES results with CASES-99 observations}

We now present comparisons of surface layer variables $\left(u_{*}, \overline{\tilde{w}^{\prime} \tilde{\theta}_{s}^{\prime}}\right.$, and $10-\mathrm{m}$ wind speed $\left.U_{10}\right)$ obtained from LES with the corresponding C99 observations. Results for surface momentum fluxes as represented by the evolution of friction velocity $u_{*}$ are presented in Fig. $4 \mathrm{a}$. The LES results for $u_{*}$ demonstrate general agreement with the observational trends. There is reasonable quantitative agreement in daytime convective boundary layers, but there is an overestimation of surface momentum fluxes in the stable boundary layers. To be specific, $u_{*}$
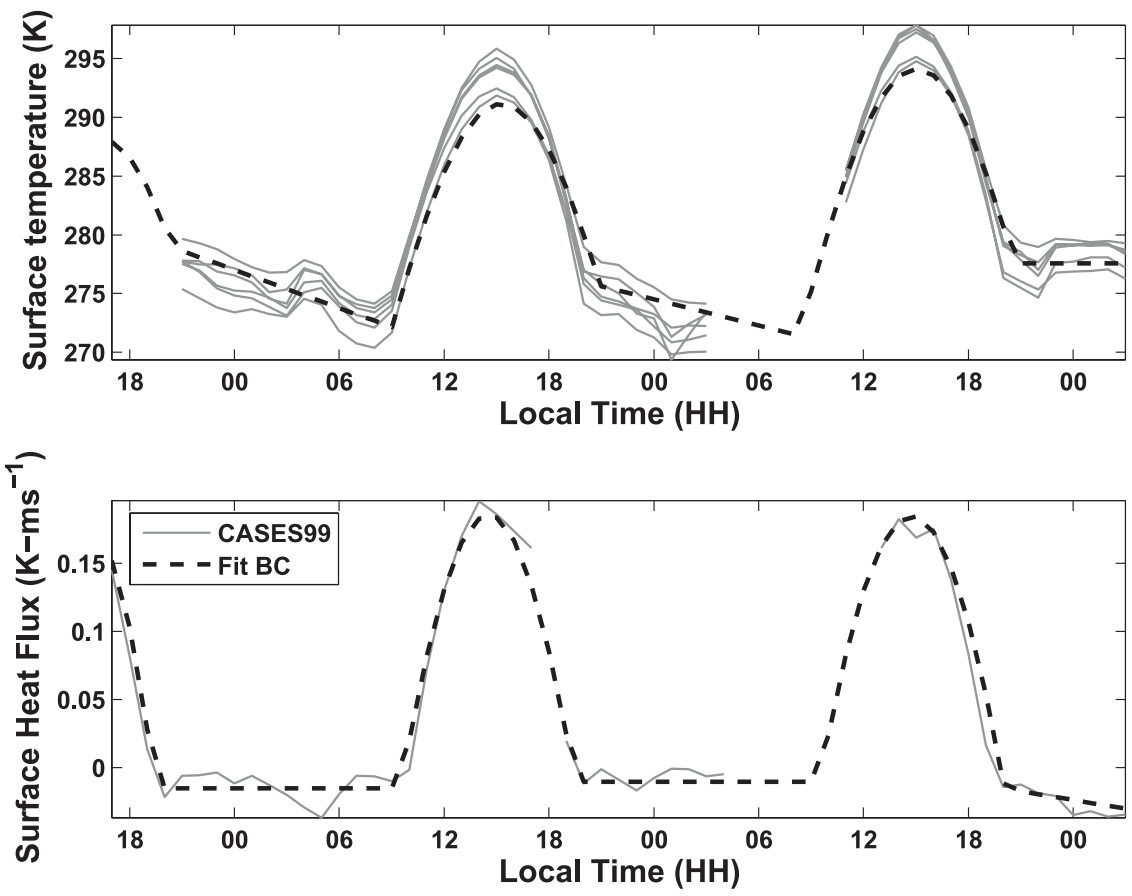

FIG. 2. Time evolution of CASES99 observations and the derived fit for implementation as LES boundary condition for the variables (top) surface temperature $T_{s}$ and (bottom) surface sensible heat flux $H$. 


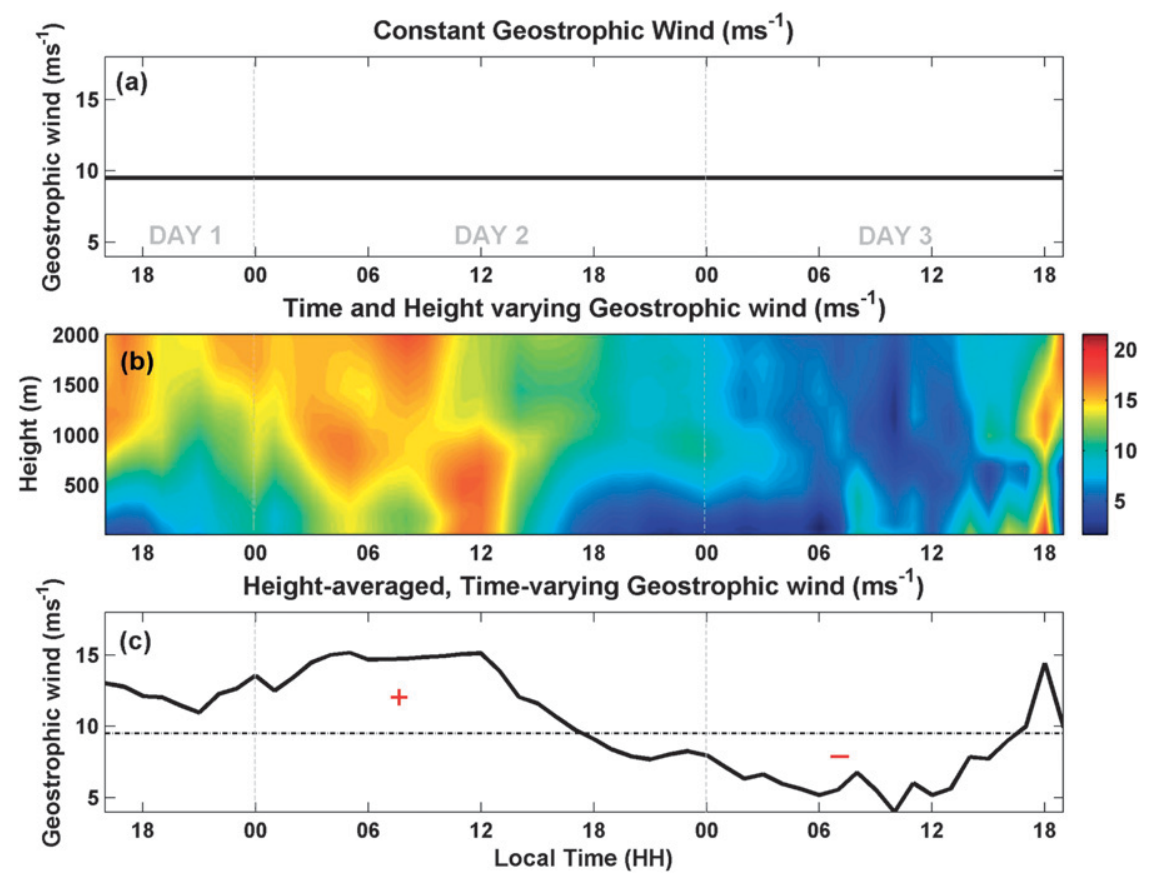

FIG. 3. The three geostrophic wind profiles used as mean pressure forcing in LES, showing the magnitude of (a) constant $\left(\left|\mathbf{G}^{\text {const }}\right|\right)$, (b) time- and height-varying $[|\mathbf{G}(z, t)|]$, and (c) heightaveraged, time-varying $[|\mathbf{G}(t)|]$ geostrophic wind conditions. The plus and minus signs in (c) indicate where the geostrophic wind is faster or slower than the $\left|\mathbf{G}^{\text {const }}\right|\left(=9.5 \mathrm{~m} \mathrm{~s}^{-1}\right)$. The vertical gray dashed lines demarcate the three days that are correspondingly referred to in the text as day 1 , day 2 , and day 3 .

does not decay to the lowest values as observed in C99 and instead remains at a nearly constant level (around $0.24 \mathrm{~m} \mathrm{~s}^{-1}$ ) for a large part of the stable regime in the two nocturnal boundary layer regimes (hours 2000-0600). The observed discrepancy can be attributed to various factors such as numerical resolution, simplified surface boundary conditions, and constant geostrophic forcing. Recent research on LES simulations of stable boundary layers (Beare and MacVean 2004; Beare et al. 2006) has emphasized the impact of numerical resolution on underestimation of momentum fluxes in the SBL. We have tried simulating the same setup at various resolutions $\left(96^{3}-160^{3}\right)$ and found that the incremental improvements at higher numerical resolutions became very small. This implies that with everything else being the same, an incremental resolution increase applied to a moderateresolution LES (as in this case) cannot eliminate the observed differences in the SBL regime. Note that this observation might be specific to our LES code, owing to use of accurate pseudospectral numerics and resolution independence characterized by dynamic SGS models.

A trend similar to $u_{*}$ is found for the time series of 10 -m horizontal wind speed $U_{10}$ (Fig. 4b). However, while the $u_{*}$ observations clearly demarcate the unstable and stable regimes, the same level of distinction is not visible with the $U_{10}$ observations. Notice that when compared with the agreement exhibited by $u_{*}, U_{10}$ results achieve better quantitative agreement with the C99 observations during the first $22 \mathrm{~h}$ (2000 day 1-1800 day 2). However, the wind speed observations are strongly underestimated by the LES results in the subsequent stable regime (1800 h day $2-0800 \mathrm{~h}$ day 3 ), similar to the behavior exhibited by $u_{*}$ results from LES. Another interesting observation emerges from the behavior of $U_{10}$ results in the time period $1000-1600$ day 3 . Here, the temporal trend exhibited by the LES results is exactly opposite to the trend from the C99 observations.

To understand the reasons for this behavior and the discrepancies identified above, we need to recall that the two main drivers of the LES results are the surface flux boundary condition and geostrophic forcing. Thus, the inability of LES to capture the magnitude and temporal trends of $10-\mathrm{m}$ wind speed $U_{10}$ results most likely from the nature of the surface flux boundary condition (imposed surface temperature or surface flux) and geostrophic forcing (constant with time, time varying, or time and height varying).

We also compare the behavior of surface sensible heat flux $\overline{\tilde{w}^{\prime} \tilde{\theta}_{s}^{\prime}}$ obtained from the $S_{T}^{\text {const }}$ simulation (Fig. 4c). The LES demonstrates good qualitative agreement with 
(a)

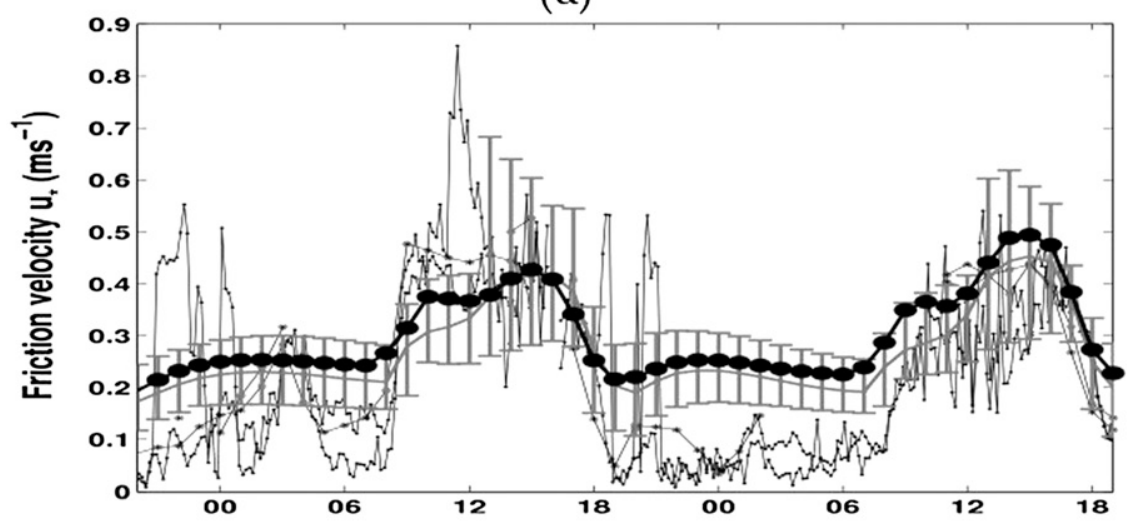

(b)

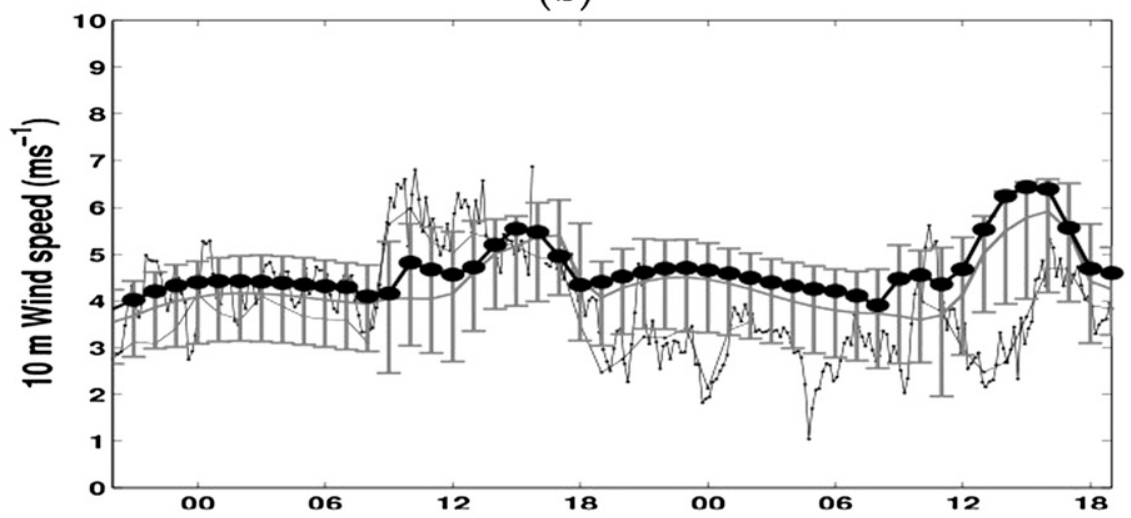

(c)

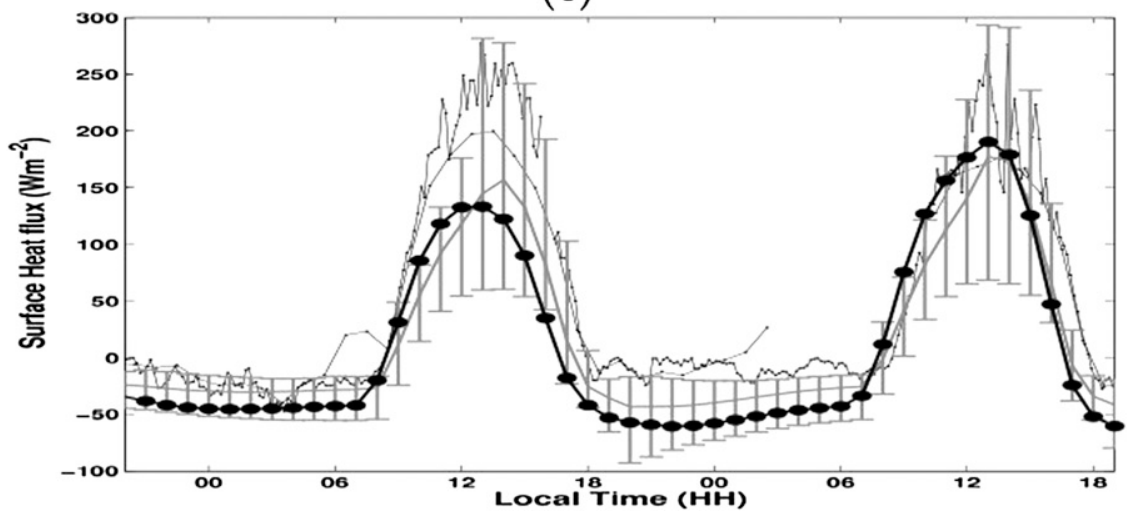

FIG. 4. Temporal evolution of (a) friction velocity $u_{*}$, (b) $10-\mathrm{m}$ wind speed $U_{10}$, and (c) sensible heat flux $\overline{w^{\prime} \theta_{s}^{\prime}}$ for the $S_{T}^{\text {const }}$ (black dotted line) LES case. The SCM intercomparison runs are shown as solid gray lines with error bars representing the spread of the various SCM codes. The C99 observations are plotted as thin black lines.

the C99 observations and captures well the transition from stable to unstable regimes at sunrise as corroborated by the overlap between the LES results and observations between 0700 and 0100 on both mornings. However, the LES results produce stronger cooling (overestimation of fluxes) during the night and weaker heating (underestimation of fluxes) during the day as compared with the observations, which could be a result of the nature of the surface flux boundary condition (based on $T_{s}$ and $\left.z_{0 h}\right)$.

To ascertain the impact of the surface flux boundary condition, an LES was also performed using an imposed heat flux $H$ obtained as a fit to the C99 observations as shown in the bottom panel of Fig. 2. The rest of the 
(a)

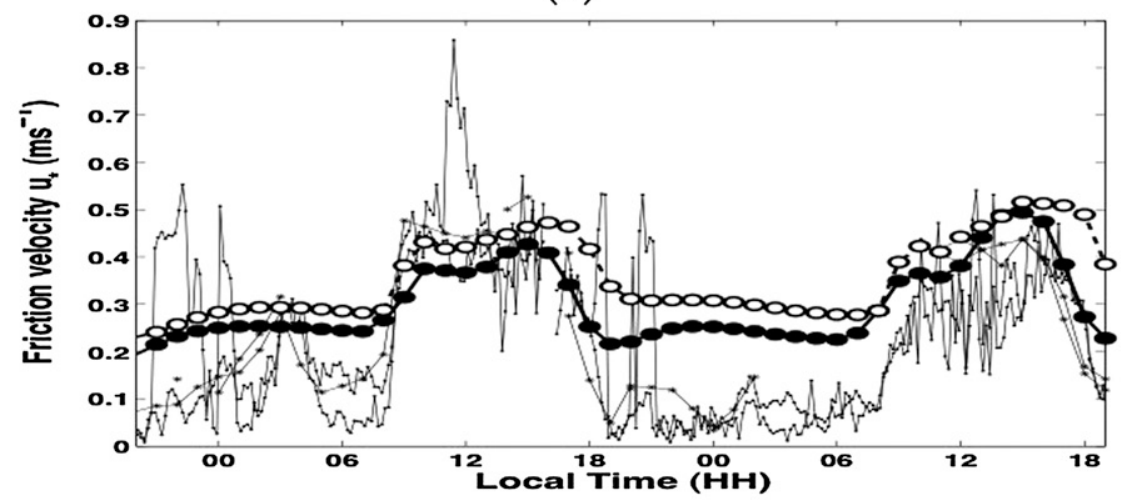

(b)

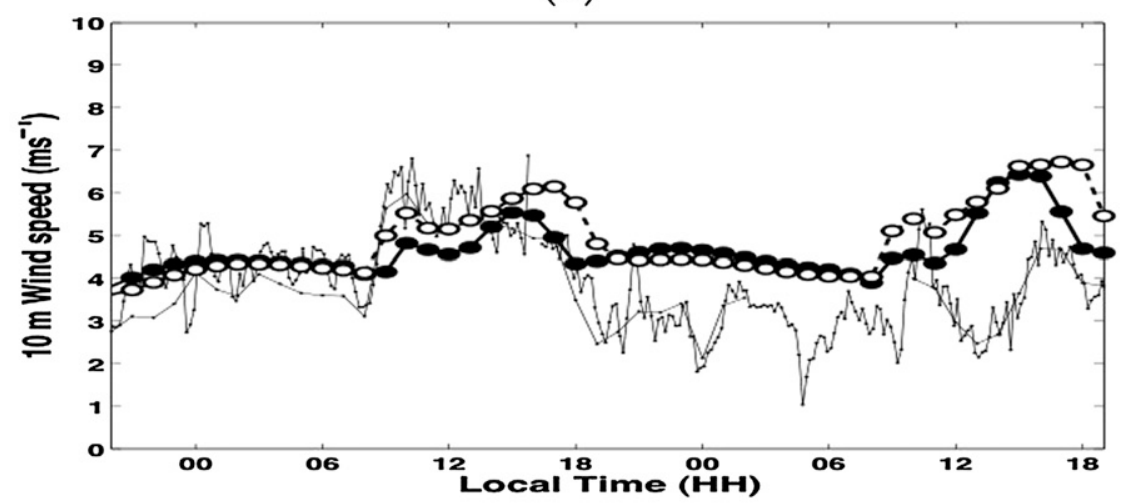

(c)

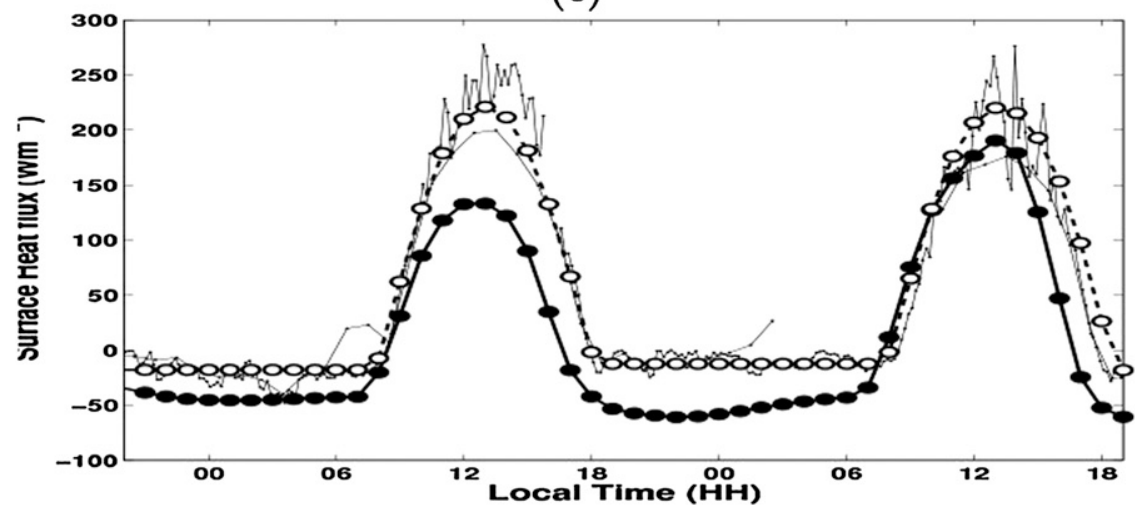

FIG. 5. Temporal evolution of (a) friction velocity $u_{*}$, (b) 10 -m wind speed $U_{10}$, and (c) sensible heat flux $\overline{w^{\prime} \theta_{s}^{\prime}}$ for the $S_{T}^{\text {const }}$ (filled circles) and $S_{H}^{\text {const }}$ (open circles) LES cases. The C99 observations are plotted as thin black lines.

simulation setup remains the same as above. We will refer to this simulation as $S_{H}^{\text {const }}$. The results from the $S_{H}^{\text {const }}$ simulation have been added to the previous simulation case with the imposed surface temperature in Figs. 5a-c. In all of the subsequent plots, results from LES cases with surface temperature $T_{s}$ and heat flux $H$ will be represented by filled and open symbols, respectively.

Note that the two surface flux boundary conditions $\left(T_{s}, H\right)$ primarily differ in the degree of surface-atmosphere coupling embedded in the surface flux estimation. The heat flux $H$ is essentially a one-way surface $\rightarrow$ atmosphere input where the imposed surface heat flux is independent of the ABL flow dynamics. The surface heat flux based on imposed surface temperature $T_{s}$ is dependent on the difference between the surface temperature and the overlying air temperature resulting in a semicoupled surface $\rightleftarrows$ atmosphere feedback. It can therefore be expected that the formulation based on the imposed surface temperature 
(a)

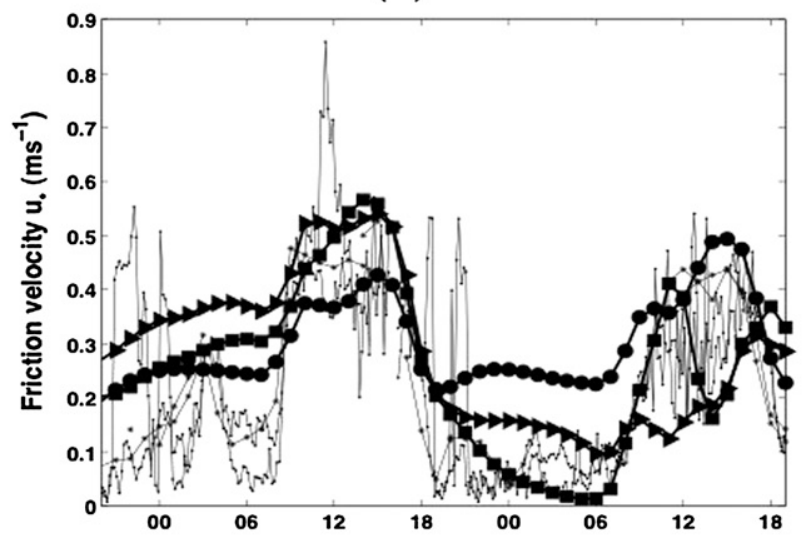

(b)

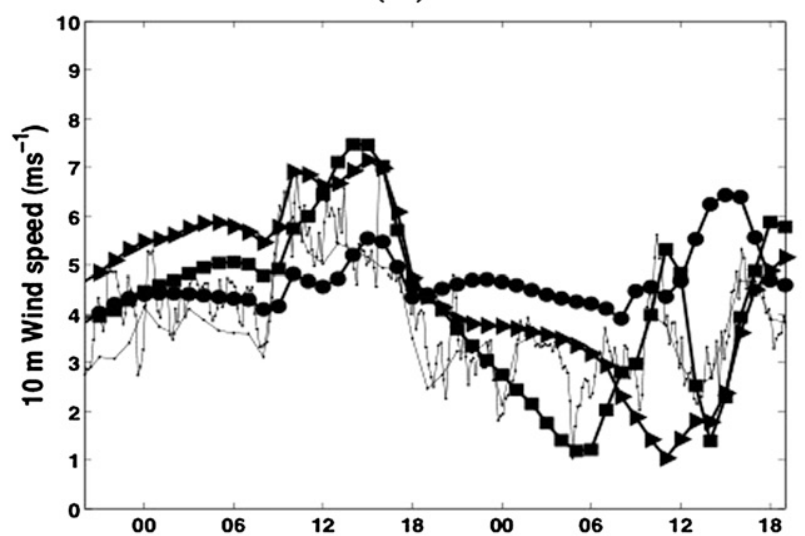

(c)

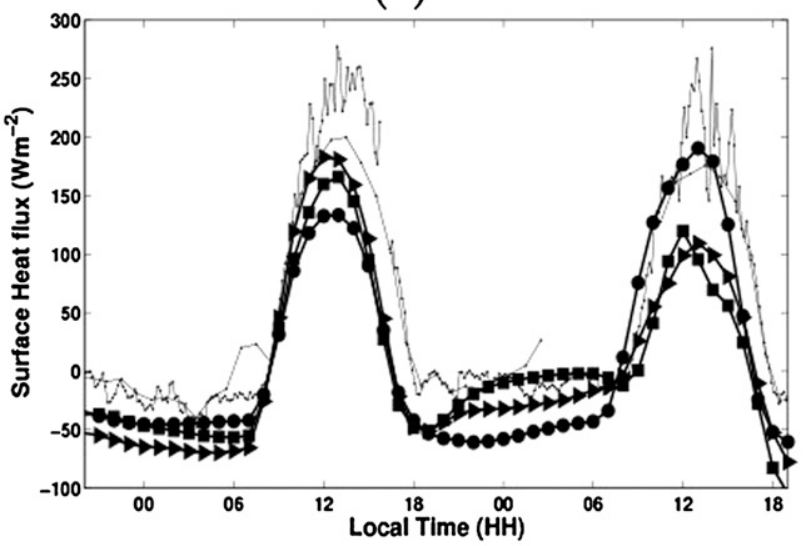

(d)

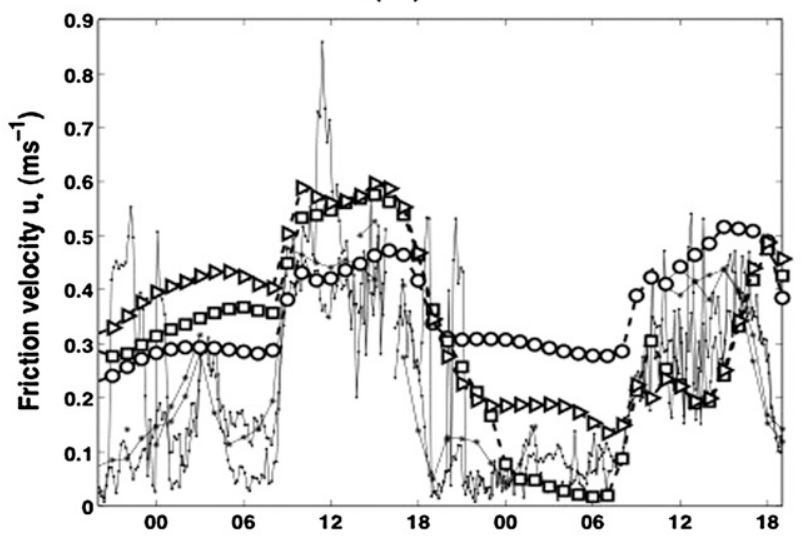

(e)

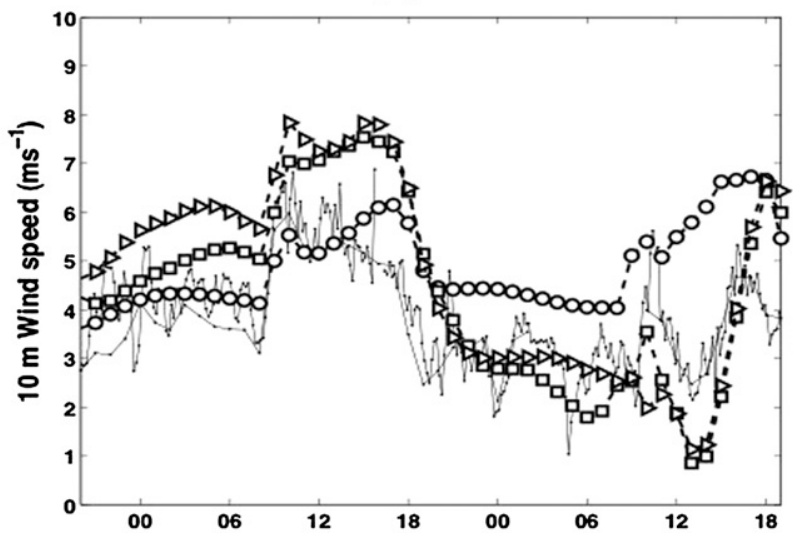

(f)

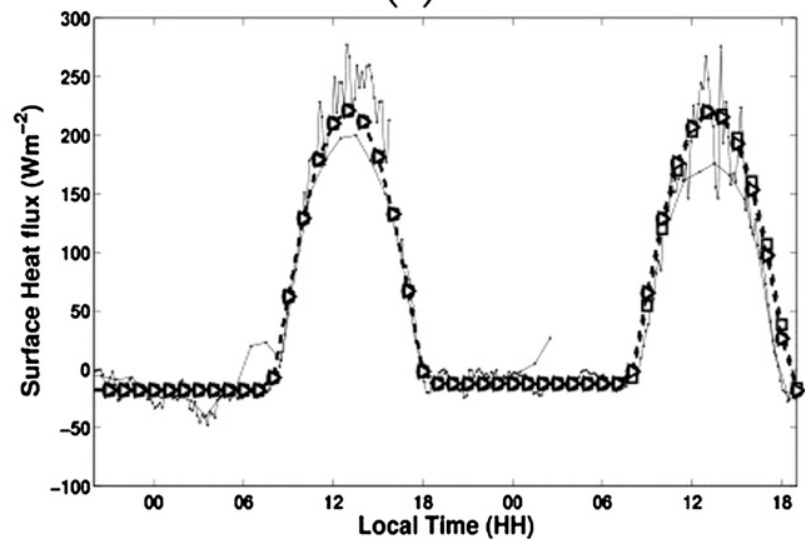

FIG. 6. Temporal evolution of friction velocity $u_{*}, 10$-m wind speed $U_{10}$, and sensible heat flux $\overline{w^{\prime} \theta_{s}^{\prime}}$ for LES simulations with (a)-(c) $T_{s}$

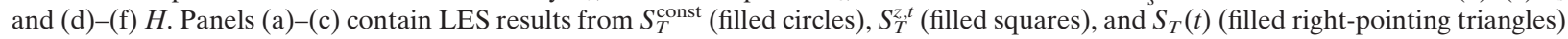
cases, and (d)-(f) represent $S_{H}^{\text {const }}$ (open circles), $S_{H}^{z, t}$ (open squares), and $S(t)$ (open right-pointing triangles) cases. The C99 observations are plotted as thin black lines.

will be more representative of the land-atmosphere interactions.

With an imposed heat flux $H\left(S_{H}^{\text {const }}\right.$ case $), u_{*}$ is consistently higher than in the LES case with imposed surface temperature $\left(S_{T}^{\text {const }}\right)$, notably in the stable boundary layer
(Fig. 5a). To be specific, the $S_{T}^{\text {const }}$ simulation yields $u_{*}$ values that are closer to the $\mathrm{C} 99$ observations in the stable boundary layer when compared with the values from the $S_{H}^{\text {const }}$ case. This indicates the importance of using a coupled surface flux condition in obtaining realistic $u_{*}$ 


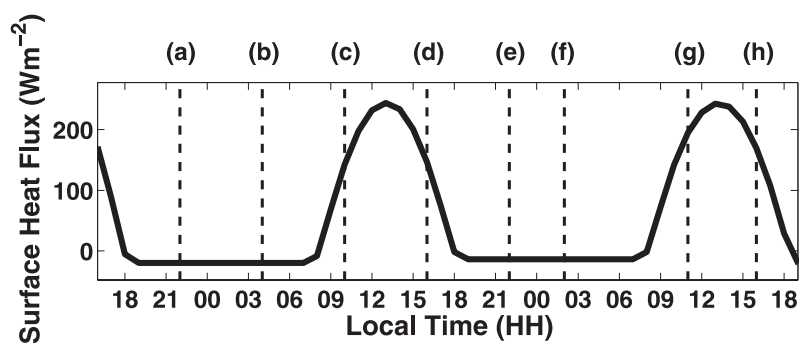

FIG. 7. Profiles at eight particular times shown here are plotted in the subsequent figures (Figs. 8-13), where the figure panel labels correspond to the time labels in this plot. The times are represented by the labeled vertical dashed lines, as follows: label a: 2200 LT 22 October, label b: 0400 LT 23 October, label c: 1000 LT 23 October, label d: 1600 LT 23 October, label e: 2200 LT 23 October, label f: 0200 LT 24 October, label g: 1100 LT 24 October, and label h: 1600 LT 24 October. The choice of the time intervals is further discussed in the text. Note that the heat flux time series serves to help to locate day and night events.

estimates from LES. In addition, the $U_{10}$ from the $S_{H}^{\text {const }}$ case is also consistently higher than the one from the $S_{T}^{\text {const }}$ case in the two daytime convective regimes (Fig. 5b), which might be caused by the corresponding higher daytime fluxes (Fig. 5c). A surprising result is that an analogous disparity in the nighttime fluxes between the two LES cases $\left(S_{T}^{\text {const }}\right.$ and $\left.S_{H}^{\text {const }}\right)$ does not influence the $U_{10}$ results similarly.

The excellent agreement of surface heat flux $\overline{\tilde{w}^{\prime} \tilde{\theta}_{s}^{\prime}}$ in the $S_{H}^{\text {const }}$ case results directly from the use of the observationderived fit as the surface flux boundary condition (Fig. 2, bottom panel). Note that in the comparative plots for the variables $u_{*}, U_{10}$, and $\overline{\tilde{w}^{\prime} \tilde{\theta}_{s}^{\prime}}$ the LES profiles from the $S_{H}^{\text {const }}$ case consistently exhibit a delayed evening transition as compared with the same profiles from the $S_{T}^{\text {const }}$ case. In other words, there is a visible time lag between the time a change in sign of heat flux occurs and a consequential impact on $u_{*}$ and $U_{10}$ profiles for the $S_{H}^{\text {const }}$ case. On the other hand, the impact of a change in sign of heat flux on $u_{*}$ and $U_{10}$ is almost immediate for the $S_{T}^{\text {const }}$ case. Recall that the imposed heat flux in the $S_{H}^{\text {const }}$ case closely matches the observed surface heat fluxes in C99. Then, why does it induce a delay in the ABL dynamics? The difference lies in the representation of land-atmosphere interaction in the LES. In reality, the measured fluxes are a product of the complex landatmosphere interactions wherein the fluxes both influence and are influenced by the ABL dynamics. In LES, specifically for the $S_{H}^{\text {const }}$ case, the measured fluxes are imposed as an external boundary condition and are "oblivious" to the evolving ABL dynamics. Therefore, the resulting evolution of variables like $u_{*}$ does not follow the same trend as in the C99 observations. The surface temperature boundary condition, on the other hand, allows for some degree of land-atmosphere interaction and is thereby more representative of real-world landatmosphere interactions.

It is evident from the comparative analysis presented above that the type of surface flux boundary condition, while strongly influencing the physics of the flow, cannot
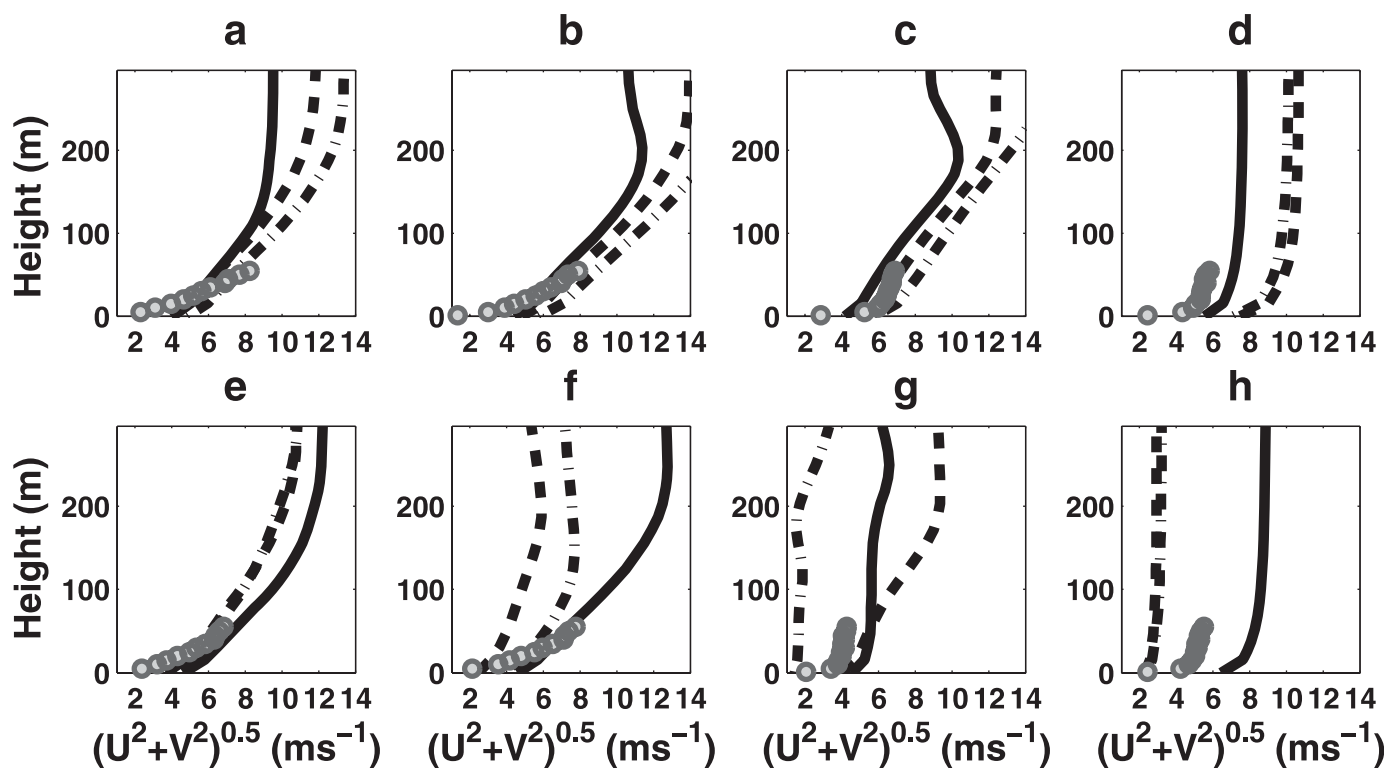

FIG. 8. Comparison of vertical profile of horizontal wind speed between LES cases with imposed surface temperature and CASES-99 observations for (a)-(h) the different times in the diurnal cycle as shown in Fig. 7. Each panel also contains profiles from the LES cases $S_{T}^{\text {const }}, S_{T}^{z, t}$, and $S_{T}^{t}$ geostrophic forcing, respectively represented by a solid line, a dashed line, and a dash-dotted line. The CASES-99 observations are represented by filled gray circles. 
a
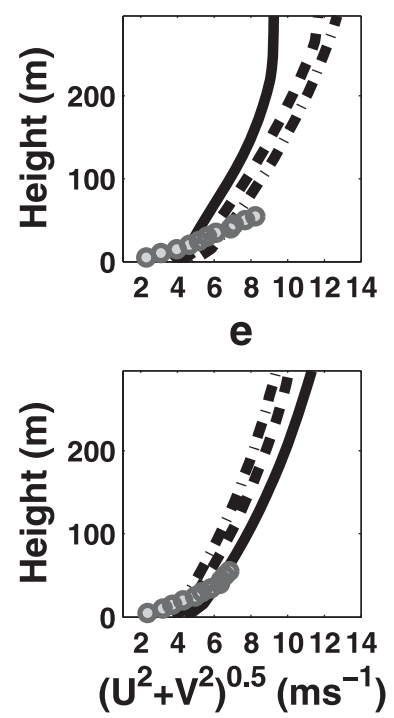

b
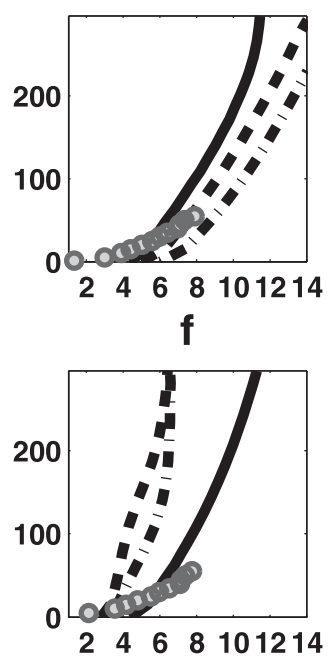

$\left(\mathrm{U}^{2}+\mathrm{V}^{2}\right)^{0.5}\left(\mathrm{~ms}^{-1}\right)$

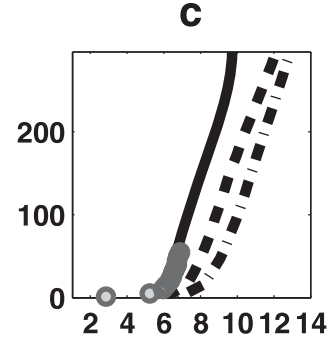

$\mathrm{g}$

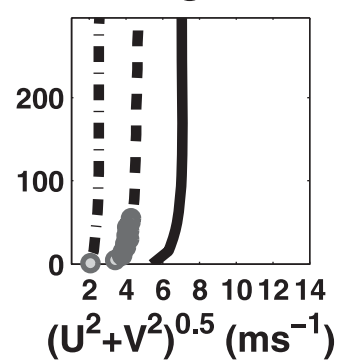

d
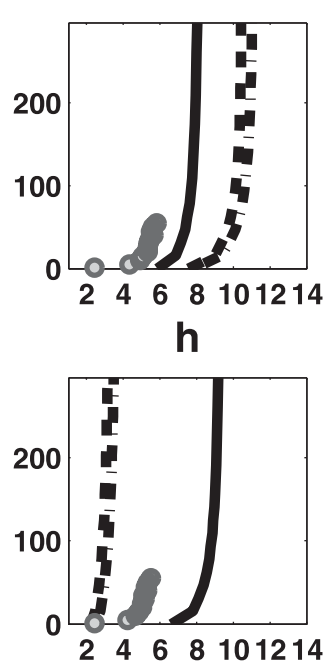

$\left(\mathrm{U}^{2}+\mathrm{V}^{2}\right)^{0.5}\left(\mathrm{~ms}^{-1}\right)$

FIG. 9. As in Fig. 8, but for LES cases with the heat flux boundary condition, and showing cases $S_{H}^{\text {const }}, S_{H}^{z, t}$, and $S_{H}^{t}$.

single-handedly account for the qualitative and quantitative differences between LES and C99 observations. Up to this point, we have only considered simulations driven by a constant geostrophic forcing. Therefore, we now consider the impact of variable geostrophic forcing.

\section{LES with spatially and temporally varying geostrophic boundary conditions}

In section $3 b$, we found that results from two LES cases forced by a constant geostrophic wind condition fail to capture accurately the trends of the C99 observations. Note that the application of two different surface boundary conditions for surface heat flux estimation (represented by the $S_{T}^{\text {const }}$ and $S_{H}^{\text {const }}$ cases) did not completely resolve the observed differences between the LES results and C99 observations. It seems therefore that the likely source of these differences might lie in the nature of the geostrophic forcing.

We have therefore performed additional simulations using realistic geostrophic forcing with realistic temporal and vertical variation. To isolate the impact of the spatial and temporal variability induced by the new geostrophic forcing, simulations driven by a height-averaged, timevarying form of the geostrophic forcing $\mathbf{G}(t)$ were also performed in which

$$
\mathbf{G}(t)=\frac{1}{z_{i}} \int_{0}^{z_{i}} \mathbf{G}(z, t) d z=\left[U_{g}(t), V_{g}(t)\right]
$$

with $z_{i}$ being the ABL height. Figures $3 \mathrm{a}-\mathrm{c}$ show a comparison of the three geostrophic forcings, represented through their magnitudes $\left|\mathbf{G}^{\text {const }}\right|,|\mathbf{G}(z, t)|$, and $|\mathbf{G}(t)|$. As in the earlier case, the simulations were performed using both temperature- and heat flux-based surface boundary conditions. This results in a total of four new simulations: $S_{T}^{z, t}, S_{T}^{t}, S_{H}^{z, t}$, and $S_{H}^{t}$. The results obtained from these simulations are compared with the two simulations presented earlier in section $3 \mathrm{~b}$. The details of these six simulations have been summarized in Table 1. For situations in which we would need to draw inferences common to the two surface boundary conditions $\left(T_{s}\right.$ and $H$ ), we will use the nomenclature $S^{\text {const }}, S^{z, t}$, and $S^{t}$ to refer to the pair of simulations forced by the respective geostrophic forcing but with surface temperature and heat flux surface boundary conditions.

\section{a. Comparison of surface layer variables from LES and CASES-99 observations}

Figures $6 \mathrm{a}$ and $6 \mathrm{~d}$ display the temporal evolution of $u_{*}$ for all six LES cases in comparison with field observations. The LES cases using geostrophic wind forcing with realistic time and height variability [i.e., $S^{z, t} \equiv\left(S_{T}^{z, t}\right.$ and $\left.S_{H}^{z, t}\right)$ ] demonstrate better agreement with the observations starting around 1000 day 2.

Recall that both the $S^{\text {const }}$ cases strongly overestimated the friction velocity $u_{*}$ in the stable regime starting from 2000 day 2. It is therefore encouraging that the $S^{z, t}$ cases show strong agreement with the same set of observations. The evening transition on day 2 (i.e., 23 October 1999) especially helps to demonstrate the impact of the nature of geostrophic forcing. The onset of the evening transition occurs at nearly the same time in $S^{\text {const }}\left[\equiv\left(S_{T}^{\text {const }}\right.\right.$ and $\left.\left.S_{H}^{\text {const }}\right)\right], S^{z, t}$, and $S^{t}\left[\equiv\left(S_{T}^{t}\right.\right.$ and $\left.\left.S_{H}^{t}\right)\right]$ simulations. 
a
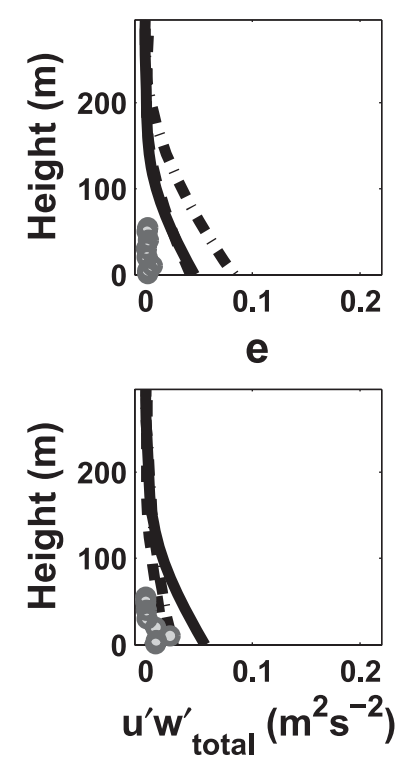

b
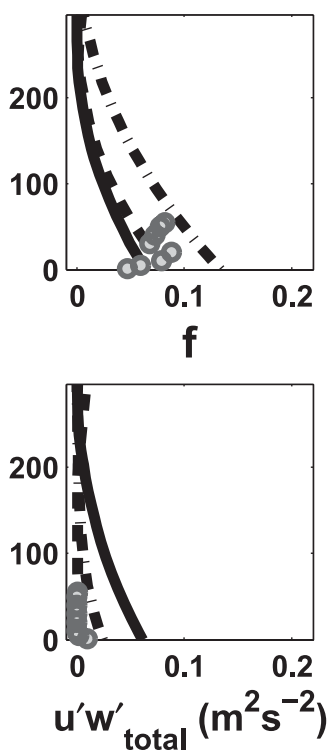

C

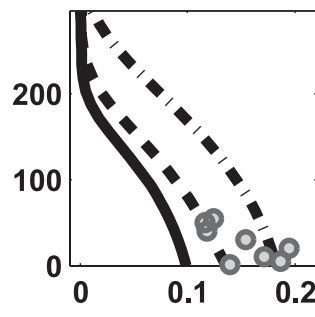

g

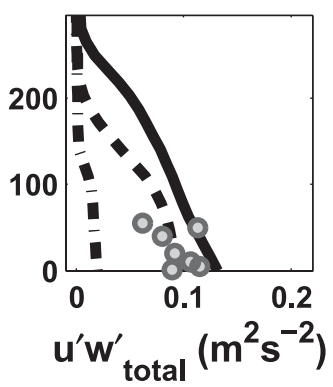

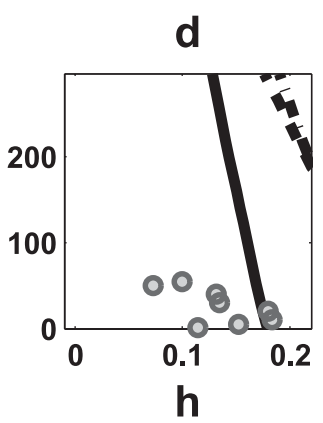

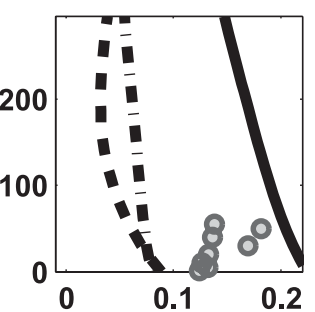

$u^{\prime} w_{\text {total }}^{\prime}\left(m^{2} s^{-2}\right)$

FIG. 10. Comparative evolution of vertical profile of total vertical momentum flux from LES cases with $T_{s}$ and CASES-99 observations. Each plot contains profiles from the LES cases $S_{T}^{\text {const }}, S_{T}^{z, t}$, and $S_{T}^{t}$ geostrophic forcing, respectively represented by a solid line, a dashed line, and a dash-dotted line. The CASES-99 observations are represented by filled gray circles.

However, $u_{*}$ in the $S^{\text {const }}$ cases starts decaying at transition $(\sim 1500)$ and continues decaying until 1800, reverses its trend, and starts increasing, eventually to level off to a near-constant plateau. None of the other cases $\left(S^{z, t}, S^{t}\right)$ exhibit such a behavior and instead exhibit a consistent posttransition decay of $u_{*}$ with comparatively higher decay rates of $u_{*}$ in the stable regime. Such a strong decay of $u_{*}$ is consistent with the C99 observations leading to better agreement of $u_{*}$ from $S^{z, t}$ and $S^{t}$ cases with C99 observations. Note that the $S^{t}$ set of simulations does not drop to values that are as low as the $S^{z, t}$ cases during the time period $0000-0800$ day 3 . This suggests that the impact of vertical structure of geostrophic forcing is more pronounced in the stable regimes. Note that the $S^{z, t}$ and $S^{t}$ cases produce similar results in the convective regime, which indicates that the presence of strong convective mixing might reduce any influence of the vertical structure of geostrophic forcing.

The aforesaid deductions are valid irrespective of the nature of the surface flux boundary condition. If we now focus on the impact of surface flux boundary conditions (i.e., comparing Figs. 6a and d), we observe that the magnitude of $u_{*}$ in the stable boundary layer is consistently smaller for LES cases with imposed surface temperature $\left(S_{T}^{\text {const }}, S_{T}^{z, t}\right.$, and $\left.S_{T}^{t}\right)$ when compared with the LES cases with imposed surface flux $\left(S_{H}^{\text {const }}, S_{H}^{z, t}\right.$, and $\left.S_{H}^{t}\right)$. In addition, the $S_{T}^{z, t}$ case captures the sharp decay in $u_{*}$ during the evening transition while the $S_{H}^{z, t}$ case exhibits delayed adjustment to the change in boundary layer dynamics, matching the $S_{T}^{z, t}$ case only starting at 0000 day 2 . The time lag observed with imposed surface heat flux in section $3 b$ is also present in the $S_{H}^{z, t}$ and $S^{t}$ cases.

The results for $U_{10}$ show trends similar to $u_{*}$ (see Figs. $6 \mathrm{~b}, \mathrm{e})$. The time series of $U_{10}$ from the pair of $S^{z, t}$ cases $\left[\equiv\left(S_{T}^{z, t}\right.\right.$ and $\left.\left.S_{H}^{z, t}\right)\right]$ exhibits good qualitative and quantitative agreement with the C99 observations. As mentioned earlier, the results from the $S^{\text {const }}$ cases show good agreement with C99 observations during 2000-0900 day 1 but severely underestimate the values from C99 observations during the subsequent time period. On the other hand, the $U_{10}$ from the $S^{t}$ cases overestimates the observations during day 1 and achieves fair agreement with the observations at later times. The behavior of $U_{10}$ from the $S^{t}$ case vis-à-vis that from $S^{\text {const }}$ can be understood by comparing Figs. 3a and 3c. The geostrophic forcing in $S^{t}$ cases is stronger than the constant forcing in $S^{\text {const }}$ cases in the first half of the simulation duration (denoted by a plus sign) and is weaker in the second half (denoted by a minus sign). This excess/deficit in the geostrophic forcing corresponds exactly to the time periods for which $U_{10}$ from the $S^{t}$ case is more/less than $U_{10}$ from the $S^{\text {const }}$ case.

We previously mentioned the inability of the $S^{\text {const }}$ setup to capture the short-term modulations in $U_{10} \mathrm{ob}-$ served during the time period $1000-1600$ day 3 . The $U_{10}$ results from the $S^{z, t}$ case capture the short-term modulations very well, verifying the importance of using 
a
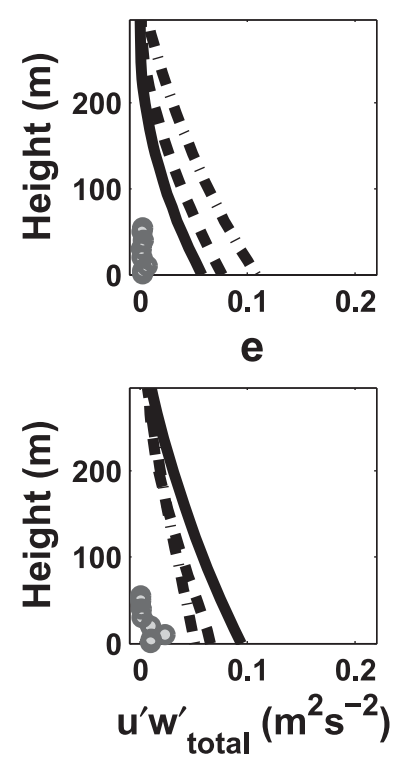

b
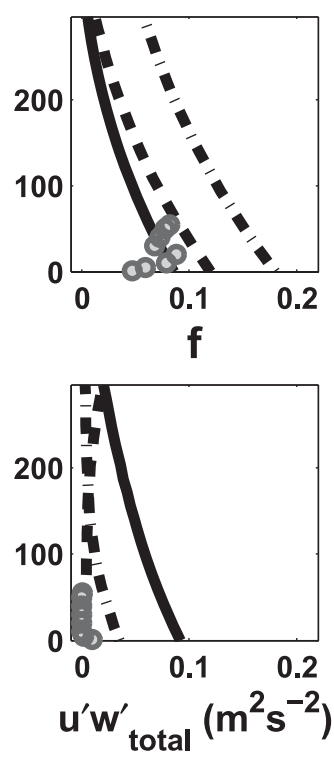

C

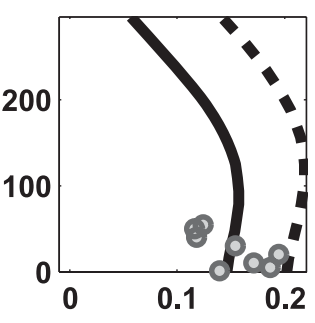

g

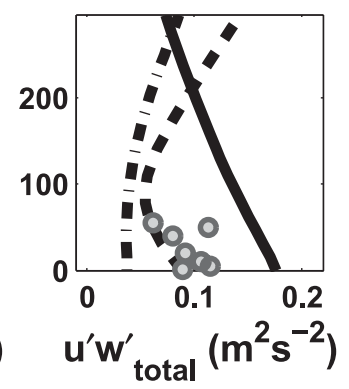

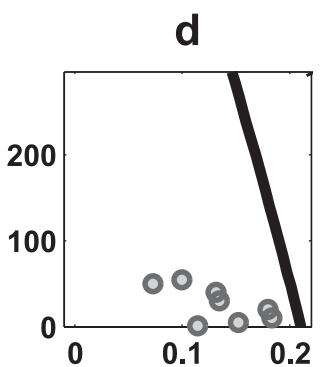

h

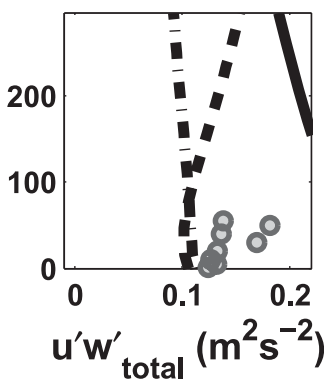

FIG. 11. As in Fig. 10, but for LES cases with the heat flux boundary condition, and showing cases $S_{H}^{\text {const }}, S_{H}^{z, t}$, and $S_{H}^{t}$.

appropriate, realistic, geostrophic forcing. In addition, it becomes clear that both the height and temporal variability of geostrophic forcing are vital to the results as the time series of $U_{10}$ from the $S^{t}$ cases do not capture the observational trends as well.

With respect to the impact of surface flux formulation, the observations are essentially the same as in the case of $u_{*}$. In short, the $10-\mathrm{m}$ wind speed $U_{10}$ values are higher with imposed heat flux $H$ than with the imposed surface temperature $T_{s}$ and have a visible time lag. The evolution of surface sensible heat flux $\overline{\tilde{w}^{\prime} \tilde{\theta}_{s}^{\prime}}$ can be seen in Figs. $6 c$ and 6f. The results from LES cases with imposed surface temperature $\left(S_{T}^{\text {const }}, S_{T}^{z, t}\right.$, and $\left.S_{T}^{t}\right)$ suffer from underestimation and overestimation of daytime and nighttime fluxes, respectively. Notice that the results from the $S_{T}^{z, t}$ case achieve good quantitative agreement with the C99 observations during the same time period of 00000600 in which $u_{*}$ also attains near-observational levels. As also mentioned in section $3 \mathrm{~b}$, the excellent agreement of the results from LES cases with imposed heat flux $H$ is expected because the heat flux presented in the plot here has been derived as a fit to the C99 observations.

The results presented in this section confirm that in addition to the nature of the surface flux boundary condition, both the magnitude and the vertical structure of geostrophic wind play a substantial role in determining the overall structure of the predicted ABL flow. The results confirm that the use of more realistic geostrophic wind forcing, such as the one derived from a mesoscale model, is beneficial in obtaining correct evolution of turbulent variables in the near-surface region. Note that a mesoscale model-derived geostrophic forcing is also likely to suffer from modeling uncertainties, which in our case might be responsible for some of the observed discrepancies. In addition, it is very likely that advection on various scales is important in this case, at least for the second half of the simulation. However, deducing advection from mesoscale models or networks of soundings is a challenging task.

So far, we have compared the performance of the six LES cases (detailed in Table 1) with respect to nearsurface observations. To validate and generalize our arguments, we now proceed to investigate the performance of these LES cases in comparison with C99 observations obtained from a vertical array of sensors.

\section{b. Comparison of LES studies with CASES-99 observations: Vertical profiles}

We first perform comparisons of vertical structure of wind speed, momentum fluxes, and sensible heat fluxes from LES cases with vertical profiles obtained from the C99 60-m main tower. Selected times, as identified in Fig. 7, were chosen for display in panels (a)-(h) of each of Figs. 8-13. The chosen times are 2200 LT 22 October, 0400 LT 23 October, 1000 LT 23 October, 1600 LT 23 October, 2200 LT 23 October, 0200 LT 24 October, 1100 LT 24 October, and 1600 LT 24 October. The choice of nonequal spacing between 2200 LT 23 October and 1100 LT 24 October has been made to ensure availability of C99 observations, because the observations are missing 

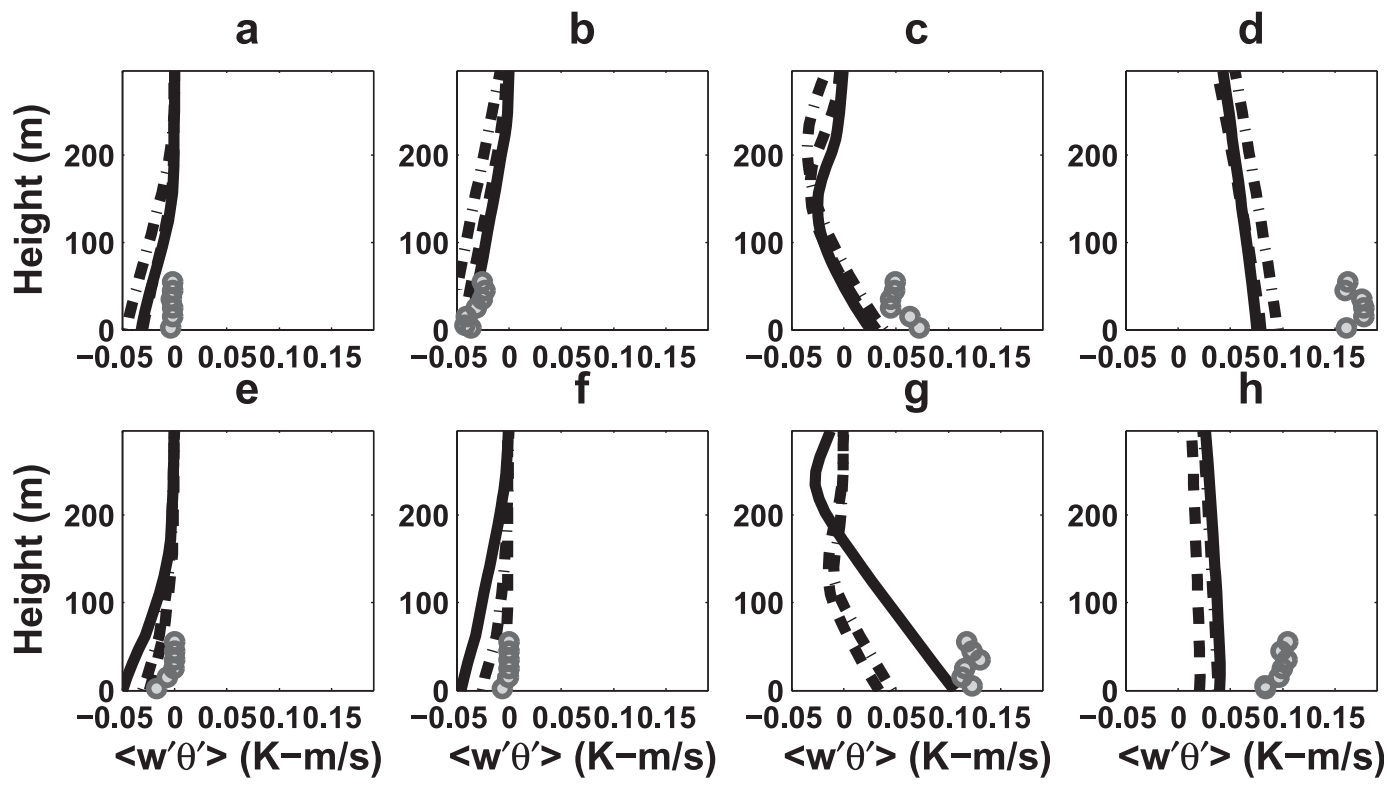

FIG. 12. Comparison of vertical profile of total sensible heat flux between LES cases with $T_{s}$ and CASES-99 observations. Each plot contains profiles from the LES cases $S_{T}^{\text {const }}, S_{T}^{z, t}$, and $S_{T}^{t}$ geostrophic forcing, respectively represented by a solid line, a dashed line, and a dash-dotted line. The CASES-99 observations are represented by filled gray circles.

from 0300 LT 24 October to 1000 LT 24 October. Figures 8, 10 , and 12 present results for wind speed, momentum fluxes, and sensible heat fluxes, respectively, for the LES cases forced with surface temperature $\left(S_{T}^{\text {const }}, S_{T}^{z, t}\right.$, and $\left.S_{T}^{t}\right)$. The same variables are presented also for the LES cases forced with imposed heat flux $\left(S_{H}^{\text {const }}, S_{H}^{z, t}\right.$, and $\left.S_{H}^{t}\right)$ in Figs. 9, 11, and 13.

The comparative profiles of horizontal wind speed are shown in Figs. 8 and 9. We first present general observations valid for both of the surface boundary condition formulations ( $T_{s}$ and $H$ ). The entire set of LES results for horizontal wind speed exhibits general qualitative agreement with the C99 observations, but the $S^{\text {const }}$ LES cases demonstrate a marginally higher agreement with the C99 observations, with the $S_{T}^{\text {const }}$ performing better than the $S_{H}^{\text {const }}$ case. Specifically, the agreement obtained by $S^{\text {const }}$ cases in Figs. 8a,b,c,e,f and 9a,b,c,e,f is very noteworthy. The profiles from the $S^{\text {const }}, S^{z, t}$, and $S^{t}$ LES cases are generally closer to each other in the nearsurface region, but they start to differ with increasing height from the surface.

A simple error estimate $\epsilon_{\alpha}$ between the LES profiles and $\mathrm{C} 99$ observations can be defined as

$$
\epsilon_{\alpha}=\left\{\frac{1}{N_{\alpha}} \sum_{z_{k}=1}^{N_{\alpha}} \frac{\left[\alpha^{\mathrm{C} 99}\left(z_{k}\right)-\alpha^{\mathrm{LES}}\left(z_{k}\right)\right]^{2}}{\alpha^{\mathrm{C} 99}\left(z_{k}\right)^{2}}\right\}^{1 / 2},
$$

where $\alpha^{\mathrm{C} 99}$ and $\alpha^{\mathrm{LES}}$ represent the chosen variable from CASES-99 and LES, respectively, and $\alpha\left(z_{k}\right)$ represents the particular grid point $z_{k}$ (of a total of $N_{\alpha}$ points) in the interval $0-55 \mathrm{~m}$. During nighttime, the fluxes drop to small values, which, combined with the noisy character of the vertical profiles, can therefore result in large error estimates due to the normalization process in Eq. (10). Therefore, to show both nighttime and daytime error estimates, we have adopted the usage of the $\log _{10}$ scale for the $y$ axis (showing the error estimate $\epsilon_{\alpha}$ ) in Figs. 14 and 15.

The temporal evolution of the error for wind speed $\epsilon_{U}$ is shown in Figs. 14 and 15 for the $S^{\text {const }}, S^{z, t}$, and $S^{t}$ pair of LES cases. The $S^{\text {const }}$ case generally shows the smallest $\epsilon_{U}$ except in the time periods of 2000-0000 day 2 and 1100-1800 day 3, where the $S^{z, t}$ and $S^{t}$ cases perform well. With respect to the impact of the surface boundary condition, the LES cases with imposed surface temperature $\left(S_{T}^{\text {const }}, S_{T}^{z, t}\right.$, and $\left.S_{T}^{t}\right)$ yield marginally better agreement with the C99 tower observations relative to the LES cases with an imposed surface heat flux $\left(S_{H}^{\text {const }}, S_{H}^{z, t}\right.$, and $\left.S_{H}^{t}\right)$. For instance, $\epsilon_{U}$ is comparatively lower for the LES cases with surface temperature (Figs. 14a, 15a). In terms of vertical profiles, the LES cases with imposed surface temperature and surface heat flux are similar for the late afternoon times (Figs. 8d,h, 9d,h), but they differ significantly for the other times, especially in the stable regimes (Figs. 8a,b,c,e,f and 9a,b,c,e,f). Some of these differences 


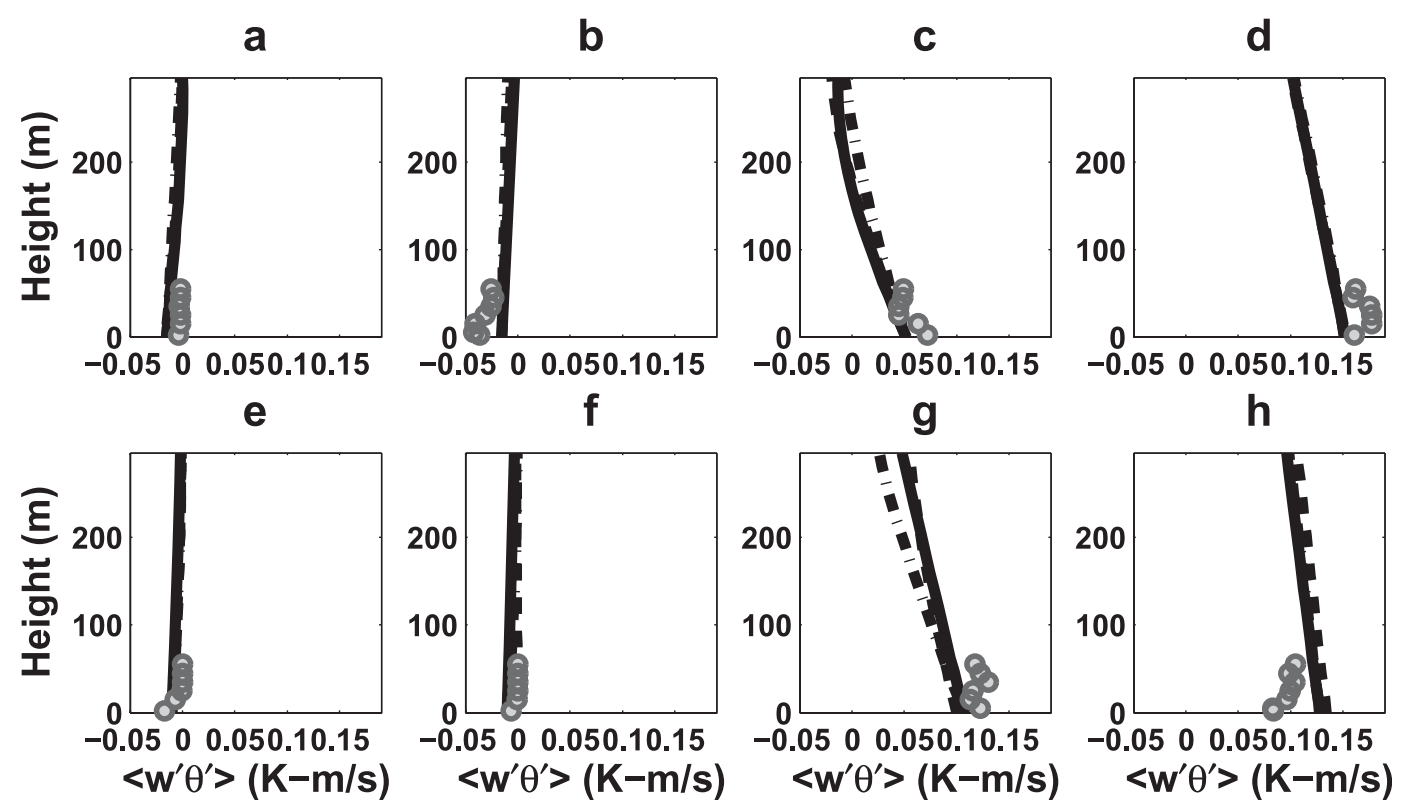

FIG. 13. As in Fig. 12, but for LES cases with heat flux boundary condition, and showing cases $S_{H}^{\text {const }}, S_{H}^{z, t}$, and $S_{H}^{t}$.

include the presence and location of the low-level jet (Figs. 8b,c and 9b,c), shape and slope of the profile (Figs. $8 \mathrm{a}, \mathrm{c}, \mathrm{f}, \mathrm{g}$ and $9 \mathrm{a}, \mathrm{c}, \mathrm{f}, \mathrm{g})$, and so on.

Moving to comparisons of momentum fluxes, we note that both the $S_{T}^{\text {const }}$ and $S_{T}^{t}$ cases show a fair degree of agreement with the C99 tower profiles. However, we find that the $S^{z, t}$ case shows good agreement with the C99 profiles, notably in the second stable regime (i.e., Figs. $10 \mathrm{c}, \mathrm{e}, \mathrm{f}, \mathrm{g})$. This was also evident in the evolution of $u_{*}$ (which is a measure of the momentum fluxes at the surface) as seen in Figs. 6a and 6d. This indicates that application of a realistic geostrophic forcing in tandem with an imposed surface temperature $T_{S}$ as an LES simulation setup $\left(S_{T}^{z, t}\right.$ and $\left.S_{T}^{t}\right)$ allows LES to generate smaller values of surface momentum fluxes and thereby achieve better agreement with the observations in the stable boundary layer. This is further reinforced by comparing Fig. 10 (LES cases with imposed surface temperature) with Fig. 11 (LES cases with imposed heat flux). The LES profiles from cases with the heat flux boundary condition consistently overestimate the fluxes and show comparatively poor agreement during the stable regimes. This further reinforces our finding that the surface temperature boundary condition is probably the most appropriate for simulations of stable boundary layers (see also Holtslag et al. 2007; Basu et al. 2008).

From the temporal evolution of $\epsilon_{\left\langle u^{\prime} w^{\prime}\right\rangle}$ in Fig. 14b, it is clear that both the $S_{T}^{\text {const }}$ and $S_{T}^{z, t}$ cases show small errors while the $S^{t}$ cases yield the highest value of $\epsilon_{\left\langle u^{\prime} w^{\prime}\right\rangle}$ for a high percentage of the simulation period. The $S_{T}^{\text {const }}$ case shows smaller error than the $S_{T}^{z, t}$ cases in the first convective regime (0600-1800 day 2), and the $S_{T}^{z, t}$ case exhibits smaller error in the second stable regime (2100 0300 day 2) and the second convective regime (12001800 day 3). Note that the error $\epsilon_{\left\langle u^{\prime} w^{\prime}\right\rangle}$ from LES cases with imposed heat flux (as shown in Fig. 15b) is generally higher than that for the LES cases with imposed surface temperature, again supporting our conclusion about the superiority of the temperature boundary condition.

The profiles for total sensible heat flux $\overline{\tilde{w}^{\prime} \tilde{\theta}^{\prime}}$ (Figs. $12,13)$ exhibit nearly the same characteristics as the momentum flux profiles. In addition, the profiles from the LES cases with imposed surface temperature exhibit good agreement with the C99 tower profiles. Some of the LES profiles also match the shapes of the C99 vertical profiles very well (e.g., Figs. 12b,c,e,f). In terms of the error $\epsilon_{\left\langle w^{\prime} \theta^{\prime}\right\rangle}$ obtained from the three LES cases using imposed surface temperature $\left(S_{T}^{\text {const }}, S_{T}^{z, t}\right.$, and $\left.S_{T}^{t}\right)$, both the $S_{T}^{\text {const }}$ and $S_{T}^{z, t}$ cases generally show the least error (see Fig. 14c). Note, however, that the $S_{T}^{z, t}$ case shows high values of error in the time period of 1200-1900 day 3 whereas the $S_{T}^{\text {const }}$ case shows high error during the stable regime on day 2 . Also, the $S_{T}^{t}$ case exhibits higher error than the $S_{T}^{z, t}$ and $S_{T}^{\text {const }}$ cases for most of the simulation period.

When comparing LES cases with surface temperature with the imposed heat flux-based LES cases, we find that the shape of the vertical profiles of sensible heat flux from LES cases with imposed temperature (Fig. 12) is significantly different from the corresponding profiles produced by LES cases with imposed heat flux (Fig. 13). These differences are especially apparent in the morning 

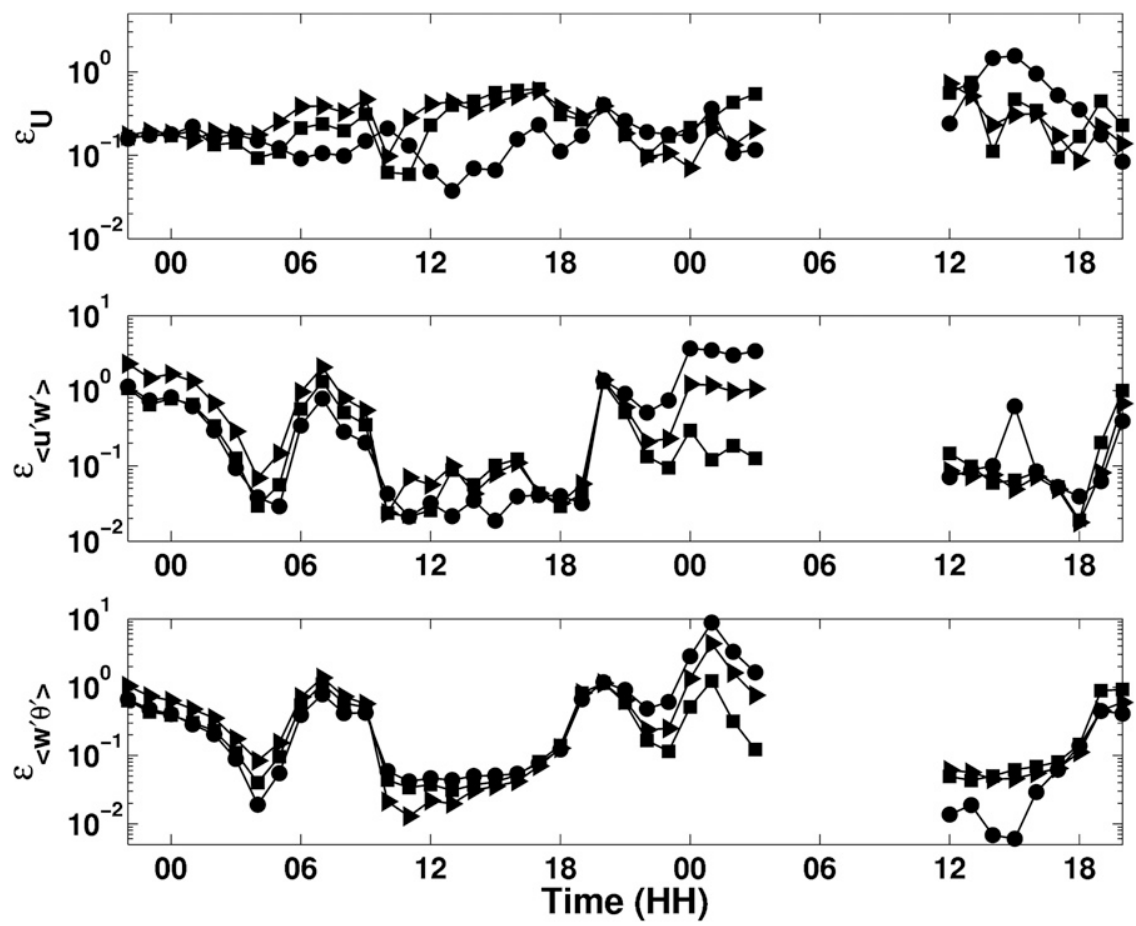

FIG. 14. Error estimates $\epsilon$ between selected variables from LES cases with imposed surface temperature and C99 observations: (a) wind speed, (b) momentum flux, and (c) sensible heat flux. Note that the $y$ axis is plotted in $\log _{10}$ scale to allow the errors of different magnitudes to be seen simultaneously. In each panel, results from the LES cases $S_{T}^{\text {const }}$ (filled circles), $S_{T}^{z, t}$ (filled squares), and $S_{T}(t)$ (filled right-pointing triangles) are shown.

(Figs. 12c,g and 13c,g) and night (Figs. 12a,e and 13a,e) profiles. Also note that, unlike the profiles of momentum flux and mean wind speed, there is a greater overlap between the heat flux vertical profiles from LES cases with imposed heat flux $\left(S_{H}^{\text {const }}, S_{H}^{z, t}\right.$, and $\left.S^{t}\right)$ with the C99 tower profiles (see also $\epsilon_{\left\langle w^{\prime} \theta^{\prime}\right\rangle}$ in Fig. 14c). The observed overlap of the LES profiles in the lower $250 \mathrm{~m}$ points to a possible unifying effect of the same surface heat flux. This possibility is further reinforced by the fact that different surface fluxes obtained with the three LES cases based on imposed surface temperature (as illustrated in Fig. 6c) result in nonoverlapping vertical profiles. A similar observation can be seen from the corresponding $\epsilon_{\left\langle w^{\prime} \theta^{\prime}\right\rangle}$ plots (Figs. 14c, 15c).

The discussion presented above, however, still leaves unresolved the question regarding the performance over the entire atmospheric boundary layer. As it turns out, using a vertical plotting scale larger than the vertical extent of C99 tower observations in Figs. 8-13 leads to an important observation. An overlap/agreement in the lower $55 \mathrm{~m}$ does not imply the same behavior at greater heights. This is clearly the case for nearly all the panels in Figs. 8-13. As noted earlier, the disparity among the different LES cases stems primarily from the temporal and vertical structure of geostrophic forcing. For instance, the geostrophic forcing for $S^{z, t}$ cases exhibits significant variability in the vertical direction (see Fig. 3b). This disparity in geostrophic forcing for the six LES cases directly impacts the temporal and vertical evolution of wind speed in the entire ABL as can be seen from Figs. 16 and 17 . The ABL dynamics as represented by the wind speed evolution are confined to the lowest $1 \mathrm{~km}$ with the $S^{\text {const }}$ cases. On the other hand, the temporal and vertical structure of geostrophic forcing results in an effect on the entire vertical domain with $S^{t}$ and $S^{z, t}$. The $S^{\text {const }}$ cases show a weak LLJ at lower heights as compared with a stronger LLJ at a higher level with the $S^{t}$ case. The wind speed in the $S^{z, t}$ case, on the other hand, has been strongly influenced by the vertical structure of geostrophic forcing, which has resulted in total elimination of the LLJ.

With respect to the surface boundary condition formulation, note that the LES cases with the imposed surface temperature produce shallower boundary layers in both convective and stable regimes as well as exhibit slower posttransition growth rates of both convective and stable boundary layers. While this observation can be verified by visual examination of any two panels from 

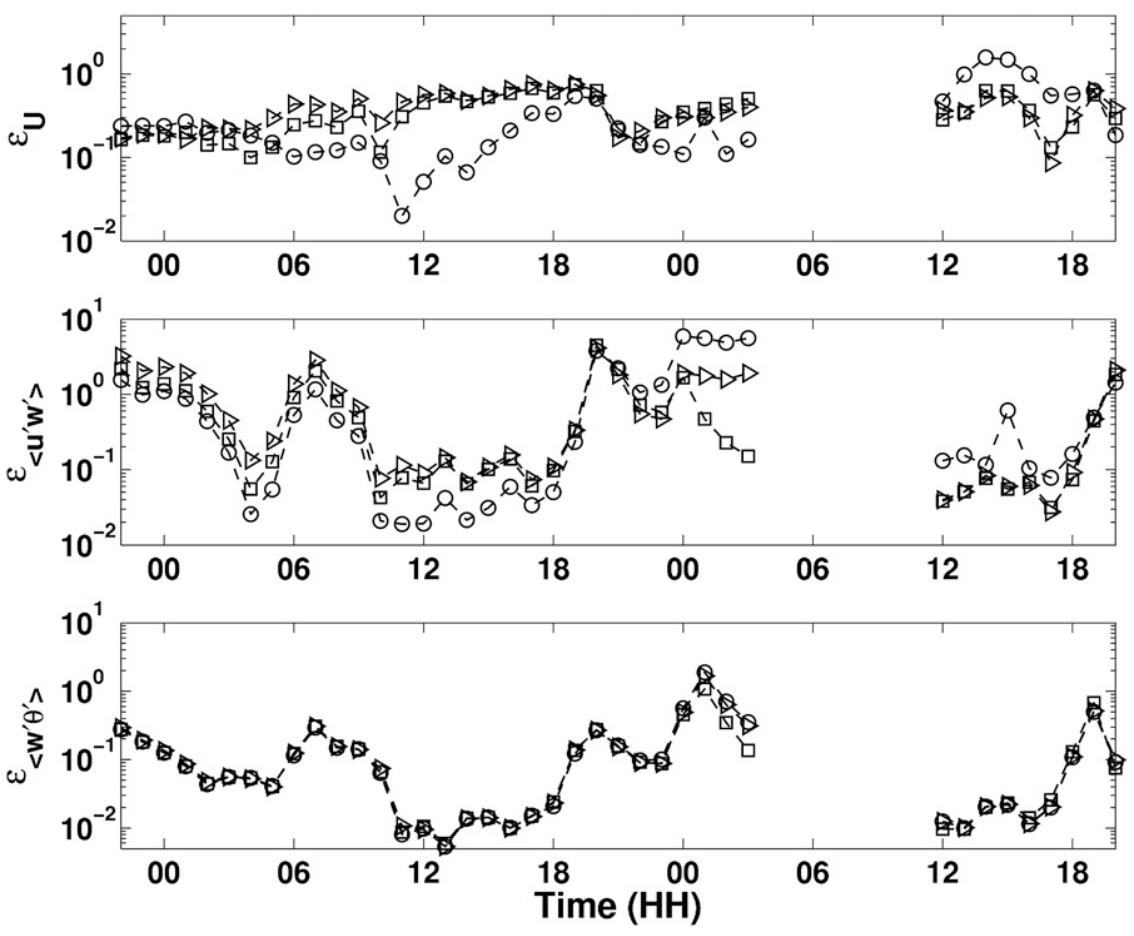

FIG. 15. As inFig. 14, but for imposed surface heat flux and C99 observations, and showing cases $S_{H}^{\text {const }}$ (open circles), $S_{H}^{z, t}$ (open squares), and $S(t)$ (open right-pointing triangles).

Figs. 16 and 17, we have also verified this by computing the temporal evolution of boundary layer height (not shown).

Last, to assess if the temporal and entire vertical structure obtained through LES is realistic, we used vertical soundings during C99 to construct an interpolated representation of temporal and vertical structure of the entire ABL. This is shown in Fig. 18. Although the results from the two $S^{\text {const }}$ cases do not show agreement with the soundings, they still demonstrate well-known features of the diurnal ABL such as nocturnal low-level jets and the characteristic mixed layer velocity profiles. Note that this lack of agreement with the $S^{\text {const }}$ LES cases is a direct consequence of the simple constant geostrophic forcing. The temporal and vertical structure of wind speed from the two $S^{z, t}$ cases exhibits good qualitative agreement with the soundings. This confirms the impact of appropriate geostrophic forcing and surface boundary conditions in accurately reproducing the observational state of the ABL. However, there are still some discrepancies such as in the lower $800 \mathrm{~m}$ during 0000-1200 day 1 in which the simulated higher magnitudes of wind speed might have a direct bearing on overestimation of friction velocity as seen earlier in Figs. 6a and 6d. We also observe that the $S^{t}$ LES cases perform well in following the C99 observational trends. This indicates that a reasonable degree of agreement of the structure of boundary layer winds can be obtained by just matching the temporal evolution of geostrophic forcing, which is encouraging. However, as mentioned earlier, the LES case $S_{T}^{z, t}$ achieved near-observational levels of $u_{*}$ in the "notoriously difficult" stable regimes, thus highlighting the importance of using an imposed surface temperature boundary condition and an observation-derived, height- and time-varying, geostrophic forcing.

\section{Conclusions}

LES investigations of multiday evolution of the ABL flow were performed with several combinations of surface boundary conditions (temperature and heat flux) and geostrophic forcings (constant, time varying, or time and height varying). Table 6 has been compiled to summarize the relative performance of the six simulations. Plus signs (good), zeros (fair), and minus signs (poor) have been used to grade each simulation's performance in six categories: daytime and nighttime surface fluxes, daytime and nighttime flux and wind speed profiles, and daytime and nighttime soundings. Overall, the $S_{T}^{\text {const }}$ setup achieved the best agreement with the C99 observations specifically in terms of daytime surface fluxes (Fig. 6) and daytime and nighttime profiles (Figs. 8, 10, and 12). Note however that the nighttime fluxes were overestimated by the $S_{T}^{\text {const }}$ case. The LES case $S_{T}^{z, t}$ 

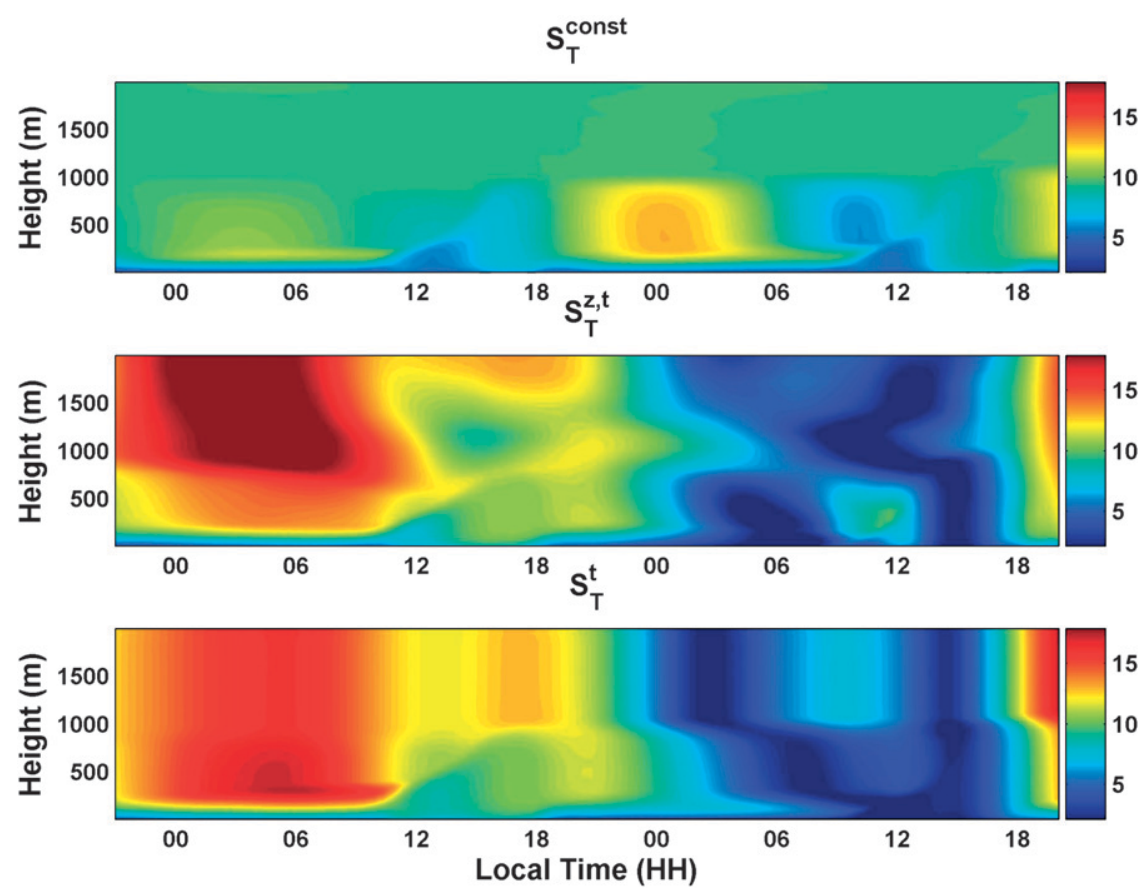

FIG. 16. Contour plots of temporal and vertical structure of wind speed from (from top to bottom) the three LES cases $\left(S_{T}^{\text {const }}, S_{T}^{z, t}\right.$, and $\left.S_{T}^{t}\right)$ with imposed surface temperature boundary condition $T_{s}$.

with the surface temperature boundary condition and driven by a realistic geostrophic forcing (with height and temporal variability) showed good agreement with the nightime fluxes on day 2. However, in comparison with the $S_{T}^{\text {const }}$ case, the $S_{T}^{z, t}$ case showed worse agreement with daytime surface fluxes and daytime and nighttime mean profiles. Both the $S_{T}^{z, t}$ and $S_{T}^{t}$ cases show good agreement with the daytime soundings. Thus, despite the more
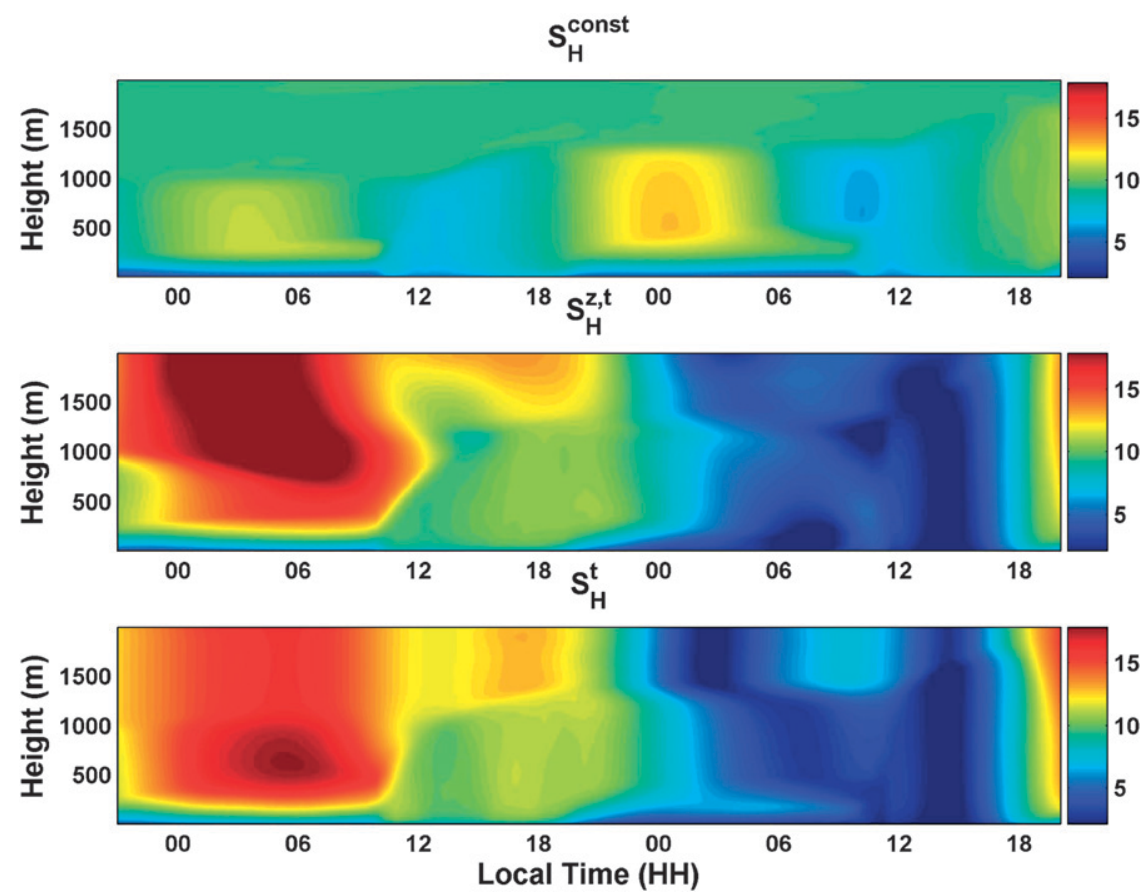

FIG. 17. As in Fig. 16, but from (top to bottom) the three LES cases $\left(S_{H}^{\text {const }}, S_{H}^{z, t}\right.$, and $S_{H}^{t}$ ) with imposed surface heat flux boundary condition $H$. 


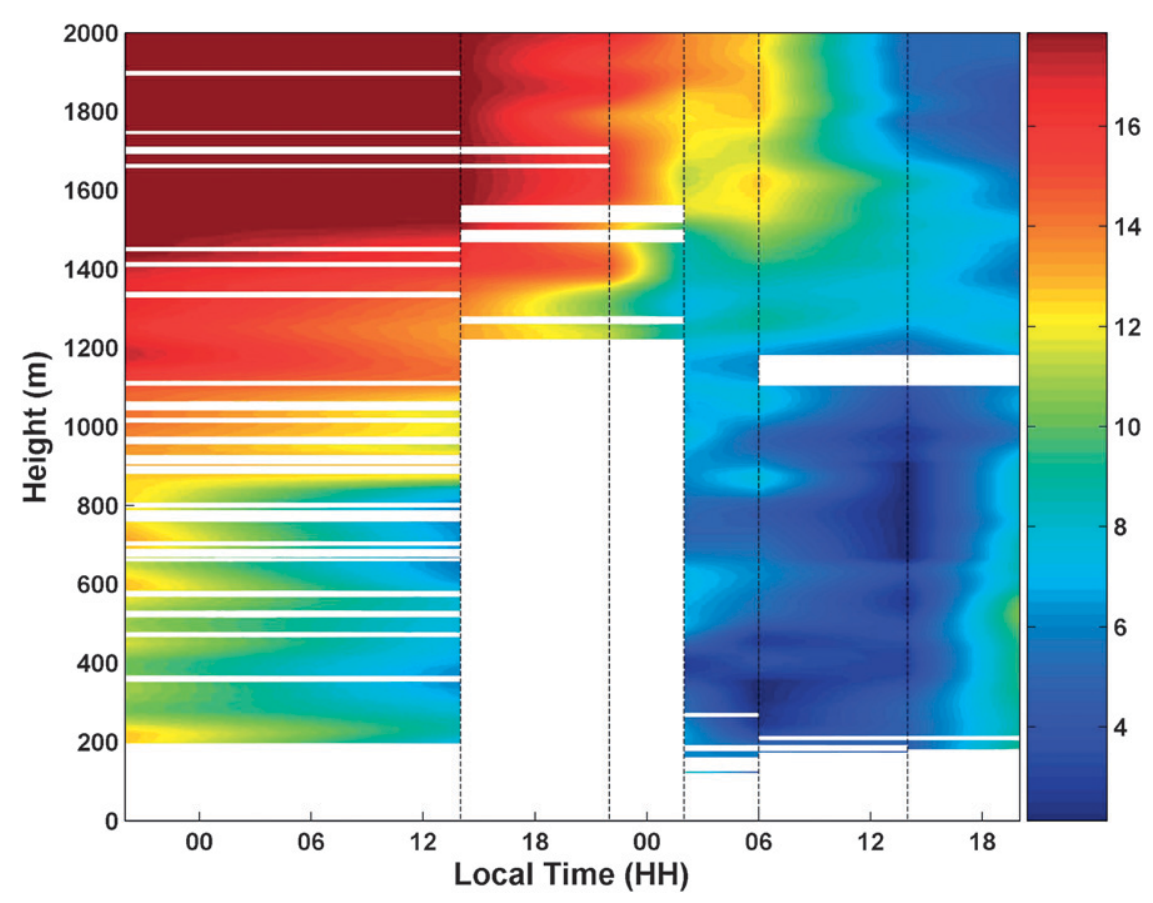

FIG. 18. Temporal and vertical distribution of wind speed obtained through linearly interpolated soundings. The vertical dashed black lines represent the times at which the soundings were obtained. The white regions represent the time periods with erroneous/missing data.

realistic geostrophic forcing, the overall agreement with the soundings leaves much to be desired, most likely because of lack of advection, which becomes more important during the second half of the simulation.

In terms of the temperature or heat flux boundary condition, a consistent set of conclusions could be reached: The LES cases with both surface temperature $\left(S_{T}^{\text {const }}, S_{T}^{z, t}\right.$, and $\left.S_{T}^{t}\right)$ and imposed heat fluxes $\left(S_{H}^{\text {const }}, S_{H}^{z, t}\right.$, and $\left.S_{H}^{t}\right)$ produced similar qualitative trends in time, the heat fluxbased cases showed poor agreement with daytime and nighttime fluxes and daytime and nighttime mean profiles. Therefore, the surface temperature boundary condition is better suited for simulations of temporally evolving ABL

TABLE 6. Performance assessment of the six LES simulations based on different combinations of surface flux formulation and geostrophic forcing. The symbols,+ 0 , and - denote good, fair, and poor agreement with the $C 99$ observations, respectively.

\begin{tabular}{lcccccc}
\hline \hline & $S_{T}^{\text {const }}$ & $S_{T}^{z, t}$ & $S_{T}^{t}$ & $S_{H}^{\text {const }}$ & $S_{H}^{z, t}$ & $S_{H}^{t}$ \\
\hline Daytime surface fluxes & + & 0 & 0 & 0 & 0 & 0 \\
Nighttime surface fluxes & 0 & + & 0 & - & 0 & 0 \\
Daytime profiles & + & - & - & 0 & - & - \\
Nighttime profiles & + & 0 & 0 & - & - & - \\
Daytime soundings & 0 & + & + & 0 & + & + \\
Nightime soundings & 0 & 0 & 0 & 0 & 0 & 0 \\
\hline
\end{tabular}

flow, as in the diurnal evolution of the ABL. We caution that although an imposed surface temperature performs relatively better than an imposed heat flux it still cannot fully reproduce the realism of land-atmosphere flux dynamics. Implementation of a surface energy balance model coupled with the LES will be the objective of future LES studies.

Acknowledgments. The authors acknowledge the financial support of The Swiss Science Foundation, CCES, the National Science Foundation (Grant NSFEAR-0609690), and the National Center for Atmospheric Research for supercomputing resources.

\section{REFERENCES}

Albertson, J. D., and M. B. Parlange, 1999a: Natural integration of scalar fluxes from complex terrain. Adv. Water Resour., 23, 239-252.

— plications for land-atmosphere interaction over complex terrain. Water Resour. Res., 35, 2121-2132.

Andrén, A., A. R. Brown, J. Graf, P. J. Mason, C.-H. Moeng, F. T. M. Nieuwstadt, and U. Schumann, 1994: Large-eddy simulation of a neutrally stratified boundary layer: A comparison of four computer codes. Quart. J. Roy. Meteor. Soc., 120, $1457-1484$. 
Basu, S., and F. Porte-Agel, 2006: Large-eddy simulation of stably stratified atmospheric boundary layer turbulence: A scaledependent dynamic modeling approach. J. Atmos. Sci., 63, 2074-2091.

— A. A. M. Holtslag, B. J. H. van de Wiel, A. F. Moene, and G. J. Steeneveld, 2008: An inconvenient "truth" about using sensible heat flux as a surface boundary condition in models under stably stratified regimes. Acta Geophys., 56, 88-99.

Beare, R. J., and M. K. MacVean, 2004: Resolution sensitivity and scaling of large-eddy simulations of the stable boundary layer. Bound.-Layer Meteor., 112, 257-281.

— , and Coauthors, 2006: An intercomparison of large-eddy simulations of the stable boundary layer. Bound.-Layer Meteor., 118, 247-272.

Beljaars, A. C. M., and P. Viterbo, 1998: The role of the boundary layer in a numerical weather prediction model. Clear and Cloudy Boundary Layers, A. A. M. Holtslag and P. G. Duynkerke, Eds., Royal Netherlands Academy of Arts and Sciences, 287-304.

Bou-Zeid, E., C. Meneveau, and M. B. Parlange, 2005: A scaledependent Lagrangian dynamic model for large eddy simulation of complex turbulent flows. Phys. Fluids, 17, 025105, doi:10.1063/1.1839152.

Chamecki, M., C. Meneveau, and M. Parlange, 2009: Large eddy simulation of pollen transport in the atmospheric boundary layer. Aerosol Sci., AS432, 241-255.

Cheng, Y., and W. Brutsaert, 2005: Flux-profile relationships for wind speed and temperature in the stable atmospheric boundary layer. Bound.-Layer Meteor., 114, 519-538.

Cuxart, J., and Coauthors, 2006: Single-column model intercomparison for a stably stratified atmospheric boundary layer. Bound.-Layer Meteor., 118, 273-303.

Derbyshire, S. H., 1999: Stable boundary-layer modelling: Established approaches and beyond. Bound.-Layer Meteor., 90, 423-446.

Hicks, B. B., 1976: Wind profile relationships from the Wangara experiments. Quart. J. Roy. Meteor. Soc., 102, 535-551.

Hodur, R. M., 1997: The Naval Research Laboratory's Coupled Ocean-Atmosphere Mesoscale Prediction System (COAMPS). Mon. Wea. Rev., 125, 1414-1430.

Högström, U., 1987: Non-dimensional wind and temperature profiles in the atmospheric surface layer: A re-evaluation. Bound.Layer Meteor., 42, 55-78.

Holtslag, A. A. M., and H. A. R. De Bruin, 1988: Applied modeling of the nighttime surface energy balance over land. J. Appl. Meteor., 27, 689-704.

_ G. J. Steeneveld, and B. J. H. van de Wiel, 2007: Role of land surface temperature feedback on model performance for stable boundary layers. Bound.-Layer Meteor., 125, 361-376.

Kleissl, J., V. Kumar, C. Meneveau, and M. B. Parlange, 2006: Numerical study of dynamic Smagorinsky models in large-eddy simulation of the atmospheric boundary layer: Validation in stable and unstable conditions. Water Resour. Res., 42, W06D10, doi:10.1029/2005WR004685.

Kosović, B., and J. A. Curry, 2000: A large eddy simulation study of a quasi-steady, stably stratified atmospheric boundary layer. J. Atmos. Sci., 57, 1057-1068.

Kumar, V., J. Kleissl, C. Meneveau, and M. B. Parlange, 2006: Large-eddy simulation of a diurnal cycle of the atmospheric boundary layer: Atmospheric stability and scaling issues. Water Resour. Res., 42, W06D09, doi:10.1029/2005WR004651.

Mason, P. J., and S. H. Derbyshire, 1990: Large eddy simulation of the stably-stratified atmospheric boundary layer. Bound. Layer Meteor., 53, 117-162.

Moeng, C.-H., 1984: A large-eddy simulation model for the study of planetary boundary-layer turbulence. J. Atmos. Sci., 41, 2052-2062.

Nieuwstadt, F. T. M., P. J. Mason, C.-H. Moeng, and U. Schumann, 1991: Large-eddy simulation of the convective boundary layer: A comparison of four computer codes. Turbul. Shear Flows, 8 , 343-367.

Porté-Agel, F., C. Meneveau, and M. B. Parlange, 2000: A scaledependent dynamic model for large-eddy simulation: Application to a neutral atmospheric boundary layer. J. Fluid Mech., 415, 261-284.

Poulos, G. S., and Coauthors, 2002: CASES-99: A comprehensive investigation of the stable nocturnal boundary layer. Bull. Amer. Meteor. Soc., 83, 555-581.

Saiki, E., C.-H. Moeng, and P. Sullivan, 2000: Large-eddy simulation of the stably stratified planetary boundary layer. Bound.Layer Meteor., 95, 1-30.

Schmidt, H., and U. Schumann, 1989: Coherent structure of the convective boundary layer derived from large-eddy simulations. J. Fluid Mech., 200, 511-562.

Steeneveld, G. J., B. J. H. van de Wiel, and A. A. M. Holtslag, 2006: Modeling the evolution of the atmospheric boundary layer coupled to the land surface for three contrasting nights in CASES-99. J. Atmos. Sci., 63, 920-935.

Svensson, G., and A. A. M. Holtslag, 2006: Single column modeling of the diurnal cycle based on CASES99 data-GABLS second intercomparison project. Preprints, 17th Symp. on Boundary Layers and Turbulence, San Diego, CA, Amer. Meteor. Soc., 8.1. [Available online at http://ams.confex.com/ams/pdfpapers/110758.pdf.]

— parison project. Gewex News, No. 17, International GEWEX Project Office, Silver Spring, MD, 9-10.

Yue, W., M. Parlange, C. Meneveau, W. Zhu, R. van Hout, and J. Katz, 2007: Large-eddy simulation of plant canopy flows using plant-scale representation. Bound.-Layer Meteor., 124, 184-203.

energy budgets in a model canopy: Comparisons between LES and wind-tunnel experiments. Environ. Fluid Mech., 8, 73-95. 